\title{
HAMILTONIAN POINCARÉ GAUGE THEORY OF GRAVITATION
}

\author{
by
}

\author{
A. López-Pinto, A. Tiemblo ${ }^{(*)}$ and R. Tresguerres ${ }^{(*)}$ \\ IMAFF, Consejo Superior de Investigaciones Científicas, \\ Serrano 123, Madrid 28006, Spain
}

\begin{abstract}
We develop a Hamiltonian formalism suitable to be applied to gauge theories in the presence of Gravitation, and to Gravity itself when considered as a gauge theory. It is based on a nonlinear realization of the Poincaré group, taken as the local spacetime group of the gravitational gauge theory, with $S O(3)$ as the classification subgroup. The Wigner-like rotation induced by the nonlinear approach singularizes out the role of time and allows to deal with ordinary $S O(3)$ vectors. We apply the general results to the Einstein-Cartan action. We study the constraints and we obtain Einstein's classical equations in the extremely simple form of time evolution equations of the coframe. As a consequence of our approach, we identify the gauge-theoretical origin of the Ashtekar variables.
\end{abstract}

\section{Introduction}

The gauge approach to Gravitation ${ }^{(1)}$ constitutes a promising departing point in the search for Quantum Gravity. Contrarily to Einstein's geometrical formulation of General Relativity (GR), the gauge theories of Gravitation provide a dynamical foundation of the gravitational interaction analogous to the Yang-Mills description of the remaining forces characteristic of the standard model. Gauge theories of Gravity are based on the local realization of a given spacetime group, which gives rise to the appearance of connections playing the role of gauge fields. In virtue of this approach, one is concerned from the beginning with dynamics rather than with geometry. In fact, the usual geometrical language employed in Gravity can be understood as deriving

(*) To my wife.

(**) To my parents. 
from a particular interpretation of the fundamental underlying gauge theory, since the resulting dynamics is formaly identical to the geometry of a certain spacetime, whose structure depends on the local spacetime group chosen. One could say that the geometry of the physical world is a consequence of the gravitational gauge interactions, i.e. of the dynamics. In particular, the Riemannian geometry of GR is recovered by imposing suitable constraints on the gauge fields. However, from the viewpoint of the gauge approach, not the geometry but the interaction described by the connections constitutes the primary subject of research.

Following a number of previous proposals ${ }^{(2)}$, we have recently shown ${ }^{(3)}$ that the natural way to construct gauge theories based on local spacetime symmetries, mostly when translations are concerned, is the nonlinear coset approach due to Coleman et al. ${ }^{(4)}$, originally introduced to deal with internal groups. The nonlinear realizations solve among others the fundamental problem of defining coframes in terms of gauge fields. The vierbeine manifest themselves as the (nonlinear) connections of the translations ${ }^{(5)}$. Furthermore, we have also proven that, for any spacetime group including the Poincaré group as a subgroup, the (anholonomic) metric tensor can be fixed once and for ever to be Minkowskian, ${ }^{(5)}$ in such a way that it does not play any dynamical role. The gravitatinal force is carried exclusively by gauge fields. The curvatures appear as the corresponding field strengths.

The present state of the observational data allows to choose among various local spacetime groups, such as the affine ${ }^{(6)}$ or the Poincaré( ${ }^{(7)}$ group, when one has to decide which one will play the role of the gauge group of Gravitation. The dynamical actions constructed in terms of such groups lead to equally acceptable classical predictions which scarcely differ from those of ordinary $\mathrm{GR}^{(8)}$. Thus, for the shake of simplicity and without prejudging the final choice, we will deal with the simplest spacetime group which includes the Poincaré group as a subgroup, namely with the Poincaré group itself. The standard Poincaré Gauge Theory (PGT) constructed on it ${ }^{(7)}$ is a good candidate to become the fundamental theory of Gravitation. In fact, as far as the Einstein-Cartan action without additional quadratic terms is concerned, the EulerLagrange equations predict the vanishing of the torsion, thus being the field equations undistinguishable from those of ordinary GR. Consequently, at the classical level both theories coincide. Nevertheless, due to the wider number of original degrees of freedom of the gauge theory, the conditions of vanishing torsion leading to the Riemannian space of GR are no more given a priori, but have to be studied as constraints. Anyway, as we will see below, the structure of our constraints essentially coincides with that predicted in the non perturbative approaches of Quantum Gravity. Not only; in fact, the dynamical variables of our nonlinear description relate in a simple way with those introduced by Ashtekar ${ }^{(9)}$.

The standard formulation of the PGT is the Lagrangian one ${ }^{(7)}$. However, Dirac's standard quantization procedure ${ }^{(10,11)}$ requires to depart from a well defined Hamiltonian formalism. Most of the difficulties concerning the quantization of Gravitation originate in the lack of a rigorous Hamiltonian approach to Einstein's classical theory. 
Particularly in the context of gauge theories in so far as Gravity is involved, it seems to be a hard task to singularize out the role of time without breaking down the underlying symmetry properties. In the usual linear gauge realization of spacetime groups, the zero-indices present in any tensor valued $\mathrm{p}$-form stand for time components, so that they are covariant but not invariant under the group action. This implies that one can deal without troubles with the Lagrangian 4-dimensional formulation, in which the spacetime is considered as a whole. But as soon as one separates time by means of a standard foliation ${ }^{(12,13)}$ in order to achieve a Hamiltonian description, the explicit spacetime invariance gets lost.

This difficulty arises even in the simple case of Maxwell's theory in the presence of Gravitation. Its 4-dimensional Lagrangian version does not present any problems. But this is no longer true when we separate the spacetime into spacelike hypersurfaces disposed along a time direction. The foliation is performed with respect to a certain timelike vector field $n:=\partial_{t}-N^{A} \partial_{A}$, with $t$ as a time coordinate. In a flat space, $t$ transforms covariantly under the Lorentz group, so that the electric field strength $E$, defined from the whole $U(1)$ field strength $F:=d A$ as minus its normal part, i.e. as $E:=-n\rfloor F$, is at least a well behaved zero-component of a Lorentz tensor. But the situation changes if we consider general coordinate transformations. In this case, the electric field strength as well as $t$ are no more Lorentz objects. This feature is common to the normal part of any $\mathrm{p}$-form representing fields of the theory if the time direction is chosen to coincide with a coordinate of the underlying spacetime manifold. A possible solution to this problem consists in taking the anholonomic timelike vector $e_{0}$ instead of an arbitrary $n:=\partial_{t}-N^{A} \partial_{A}$ as the time direction. But in any case $\left.E:=-e_{0}\right\rfloor F$ remains a single component of a Lorentz tensor. Only an invariant time vector such as the proper time or similar would allow to deal with normal parts of p-forms originally defined on a 4-dimensional manifold, behaving as Poincaré scalars.

The possibility of defining such a time direction is important if we want to construct a Hamiltonian approach to Gravity with symmetry properties analogous to those of classical mechanics. Relativistic Hamiltonians are in principle not invariant but covariant under the Lorentz group. This is a consequence of the fact that the Lagrangian density decomposes as $L=\vartheta^{0} \wedge L_{\perp}$, being the normal part $\left.L_{\perp}:=e_{0}\right\rfloor L$ a Lorentz covariant 3 -form, in terms of which the Hamiltonian is defined. Notwithstanding, it will be possible to define a Hamiltonian formalism invariant under local Poincaré transformations. The key is a nonlinear realization of the Poincaré group with its subgroup $S O(3)$ taken as the classification subgroup. Such nonlinear realization induces a Wigner-like rotation ${ }^{(14)}$ of the coframe which decomposes the fourvector-valued tetrad $\vartheta^{\alpha}$ into an $S O(3)$ threevector-valued triad $\vartheta^{a}$ plus an $S O(3)$ scalar $\vartheta^{0}$. The latter defines an invariant time in terms of which an invariant foliation of spacetime can be performed. As a result, we will have at our disposal a Poincaré invariant formalism expressed in terms of ordinary $S O(3)$ tensors and connections. When applied to Gravity, Einstein's equations simplify enormously.

The paper is organized as follows. In section 2 we introduce the nonlinear realization of the Poincaré group giving rise to the invariant time 1 -form $\vartheta^{0}$, and 
accordingly we define the new $S O(3)$ gravitational variables. Section 3 is devoted to the Poincaré invariant foliation of spacetime. In section 4 the Hamiltonian formalism is developped, and in section 5 it is applied to Yang-Mills theories in the presence of Gravity. Then, in section 6, we generalize the Hamiltonian approach to Gravitation itself. We study the Einstein-Cartan action as a relevant example, since it leads to the same Lagrangian field equations as ordinary GR. We calculate the constraints and the Hamiltonian evolution equations. In section 7 we show that they imply the Lagrangian ones, and in section 8 we present them in a more suitable $S O(3)$ formulation which reveals that the Hamiltonian equations are more restrictive than the Lagrangian ones. Finally, the relation to Ashtekar variables is pointed out in section 9. The Appendices will be useful for the readers interested in the technical details.

\section{Nonlinear gauge approach to the Poincaré group}

Let us consider the Poincaré group $G=P$, with the Lorentz generators $L_{\alpha \beta}$ and the translational generators $P_{\alpha}(\alpha, \beta=0, \ldots 3)$, satisfying the usual commutation relations as given in (B.1). We want to develop a gauge theory of this group presenting the features of a Hamiltonian formalism capable to predict the time evolution of physical systems locally defined on 3-dimensional spacelike hypersurfaces. In order to do it, we realize the Poincaré group nonlinearly with its subgroup $H=S O(3)$ as the classification subgroup, see Appendix A. This choice of the classification subgroup automatically leads to the decomposition of the fourvector-valued coframes of the standard approaches $^{(7)}$ into an $S O(3)$ triplet plus an $S O(3)$ singlet respectively in analogy to a Wigner rotation ${ }^{(14)}$-, the singlet characterizing the time component of the coframe. Needles to say that the nonlinear framework guarantees that the resulting theory posseses the whole local Poincaré symmetry, in spite of the fact that only the classification subgroup $S O(3)$ manifests itself explicitly. Thus, the invariance of the time component of the coframe under $S O(3)$ transformations means in fact Poincaré invariance. We follow the general procedure outlined in Appendix A.

We fix the anholonomic invariant metric to have the Lorentzian signature

$$
o_{\alpha \beta}:=\operatorname{diag}(-+++)
$$

and we decompose the Lorentz generators into boosts $K_{a}$ and space rotations $S_{a}$, respectively defined as

$$
K_{a}:=2 L_{a 0} \quad, \quad S_{a}:=-\epsilon_{a}^{b c} L_{b c} \quad(a=1,2,3)
$$

Their commutation relations are given in (B.6). The infinitesimal group elements of the whole Poincaré group become parametrized as

$$
g=e^{i \epsilon^{\alpha} P_{\alpha}} e^{i \beta^{\alpha \beta} L_{\alpha \beta}} \approx 1+i\left(\epsilon^{0} P_{0}+\epsilon^{a} P_{a}+\xi^{a} K_{a}+\theta^{a} S_{a}\right)
$$


and those (also infinitesimal) of the classification $\mathrm{SO}(3)$ subgroup are taken to be

$$
h=e^{i \Theta^{a} S_{a}} \approx 1+i \boldsymbol{\Theta}^{a} S_{a} .
$$

In order to realize the Poincaré group $P$ on the coset space $P / S O(3)$, we make use of the general formula (A.2) which defines the nonlinear group action, choosing in particular for the cosets the parametrization

$$
c=e^{-i x^{\alpha} P_{\alpha}} e^{i \lambda^{a} K_{a}}
$$

where $x^{\alpha}$ and $\lambda^{a}$ are the (finite) coset parameters. Other parametrizations are possible, leading to equivalent results.

According to (A.2) cum (2.3-5), the variation of the translational parameters reads

$$
\begin{aligned}
& \delta x^{0}=-\xi^{a} x_{a}-\epsilon^{0}, \\
& \delta x^{a}=\epsilon_{b c}^{a} \theta^{b} x^{c}-\xi^{a} x^{0}-\epsilon^{a},
\end{aligned}
$$

playing these parameters the role of coordinates. On the other hand, we obtain the variations of the boost parameters of (2.5) as

$$
\delta \lambda^{a}=\epsilon_{b c}^{a} \theta^{b} \lambda^{c}+\xi^{a}|\lambda| \operatorname{coth}|\lambda|+\frac{\lambda^{a} \lambda_{b} \xi^{b}}{|\lambda|^{2}}(1-|\lambda| \operatorname{coth}|\lambda|)
$$

being

$$
|\lambda|:=\sqrt{\lambda_{1}^{2}+\lambda_{2}^{2}+\lambda_{3}^{2}} .
$$

The nonlinear $S O(3)$ parameter $\boldsymbol{\Theta}^{a}$ in $(2.4)$ is calculated to be

$$
\boldsymbol{\Theta}^{a}=\theta^{a}+\epsilon_{b c}^{a} \frac{\lambda^{b} \xi^{c}}{|\lambda|} \tanh \left(\frac{|\lambda|}{2}\right) .
$$

The relevance of (2.9) becomes evident by considering the action (A.3) of the Poincaré group on arbitrary fields $\psi$ of a given representation space of the $S O(3)$ group. It reads infinitesimally

$$
\delta \psi=i \boldsymbol{\Theta}^{a} \rho\left(S_{a}\right) \psi,
$$

being $\boldsymbol{\Theta}^{a}$ precisely the nonlinear $S O(3)$ parameter $(2.9)$, and $\rho\left(S_{a}\right)$ an arbitrary representation of the $S O(3)$ group. This general transformation formula shows how the whole Poincaré group projects itself throw (2.10) on the action of the classification subgroup $S O(3)$ on its representation fields.

Now we arrive at the most important quantities in a gauge theory, namely the gauge fields. We will define the suitable connection for the nonlinear gauge realization in two steps. We first introduce the ordinary linear Poincaré connection $\Omega$ in (A.4-6) as

$$
\Omega:=-i \stackrel{(T)}{\Gamma}{ }^{\alpha} P_{\alpha}-i \Omega^{\alpha \beta} L_{\alpha \beta}=-i \stackrel{(T)}{\Gamma^{0}} P_{0}-i \stackrel{(T)}{\Gamma}{ }^{a} P_{a}+i \stackrel{(K)}{\Gamma^{a}} K_{a}+i \stackrel{(S)}{\Gamma} S_{a}
$$


which includes the translational and the Lorentz contributions $\stackrel{(T)}{ }^{\alpha}$ and $\Omega^{\alpha \beta}$ respectively, decomposed in an obvious way. All components of (2.11) transform under the linear action of the group as true Poincaré connections. In terms of them, we define the nonlinear connection (A.4) as

$$
\Gamma:=c^{-1}(d+\Omega) c=-i \vartheta^{\alpha} P_{\alpha}-i \Gamma^{\alpha \beta} L_{\alpha \beta}=-i \vartheta^{0} P_{0}-i \vartheta^{a} P_{a}+i X^{a} K_{a}+i A^{a} S_{a}
$$

Contrarily to the components of (2.11), those of (2.12) are divided into two sets with very different transformation properties. On the one hand, $A^{a}$ behaves under the Poincaré group as an $S O(3)$ connection; on the other hand, the remaining components vary as $S O(3)$ tensors. In fact we find

$$
\begin{aligned}
\delta \vartheta^{0} & =0 \\
\delta \vartheta^{a} & =\epsilon_{b c}^{a} \boldsymbol{\Theta}^{b} \vartheta^{c} \\
\delta X^{a} & =\epsilon_{b c}^{a} \boldsymbol{\Theta}^{b} X^{c} \\
\delta A^{a} & =-D \boldsymbol{\Theta}^{a}:=-\left(d \boldsymbol{\Theta}^{a}+\epsilon_{b c}^{a} A^{b} \boldsymbol{\Theta}^{c}\right) .
\end{aligned}
$$

In addition, the trivial metric $\delta_{a b}$ is a natural $S O(3)$ invariant. We will use it to raise and lower the anholonomic indices, compare with the role of the Minkowski metric in Ref.(3). The translational nonlinear connections $\vartheta^{0}, \vartheta^{a}$ in (2.12) are to be identified as the 1 -form basis geometrically interpretable as the coframe or vierbein ${ }^{(5,6,7)}$, whereas the vector-valued 1 -forms $X^{a} \equiv \Gamma^{0 a}$ represent the gauge fields associated to the boosts, and $A^{a} \equiv \frac{1}{2} \epsilon_{b c}^{a} \Gamma^{b c}$ play the role of ordinary rotational connections. The astonishing fact is that, as we have repeatedly pointed out before, the time component $\vartheta^{0}$ of the coframe is invariant under local Poincaré transformations. Let us examine this fact in more detail.

The explicit form of the nonlinear translational connection components in (2.12) reads

$$
\begin{aligned}
& \vartheta^{0}:=\tilde{\vartheta}^{0} \cosh |\lambda|+\tilde{\vartheta}^{a} \frac{\lambda_{a}}{|\lambda|} \sinh |\lambda|, \\
& \vartheta^{a}:=\tilde{\vartheta}^{a}+\tilde{\vartheta}^{b} \frac{\lambda_{b} \lambda^{a}}{|\lambda|^{2}}(\cosh |\lambda|-1)+\tilde{\vartheta}^{0} \frac{\lambda^{a}}{|\lambda|} \sinh |\lambda|,
\end{aligned}
$$

being $\tilde{\vartheta}^{\alpha}$ a coframe whose components are defined as

$$
\begin{aligned}
& \tilde{\vartheta}^{0}:=\stackrel{(T)}{\Gamma}^{0}+\left(d x^{0}-\stackrel{(K)}{\Gamma^{a}} x_{a}\right) \\
& \tilde{\vartheta}^{a}:=\stackrel{(T)}{\Gamma}^{a}+\left(d x^{a}+\epsilon_{b c}^{a} \stackrel{(S)}{\Gamma}^{b} x^{c}-\stackrel{(K)}{\Gamma}^{a} x^{0}\right) .
\end{aligned}
$$


This coframe transforms as a Lorentz four-vector under local Poincaré transformations, namely as

$$
\begin{aligned}
& \delta \tilde{\vartheta}^{0}=-\xi_{a} \tilde{\vartheta}^{a} \\
& \delta \tilde{\vartheta}^{a}=\epsilon_{b c}^{a} \theta^{b} \tilde{\vartheta}^{c}-\xi^{a} \tilde{\vartheta}^{0} .
\end{aligned}
$$

In fact, the coframe (2.15) coincides with the one we would obtain by choosing the Lorentz group as the classification subgroup of the Poincaré group, compare with Ref.(3). In order to clarify the relationship between $\vartheta^{\alpha}$ and $\tilde{\vartheta}^{\alpha}$ let us consider the following 4-dimensional representation of the Lorentz generators:

$$
\begin{gathered}
\rho\left(S_{1}\right):=-i\left(\begin{array}{rrrr}
0 & 0 & 0 & 0 \\
0 & 0 & 0 & 0 \\
0 & 0 & 0 & -1 \\
0 & 0 & 1 & 0
\end{array}\right) \quad \rho\left(S_{2}\right):=-i\left(\begin{array}{rrrr}
0 & 0 & 0 & 0 \\
0 & 0 & 0 & 1 \\
0 & 0 & 0 & 0 \\
0 & -1 & 0 & 0
\end{array}\right) \quad \rho\left(S_{3}\right):=-i\left(\begin{array}{llll}
0 & 0 & 0 & 0 \\
0 & 0 & -1 & 0 \\
0 & 1 & 0 & 0 \\
0 & 0 & 0 & 0
\end{array}\right) \\
\rho\left(K_{1}\right):=i\left(\begin{array}{llll}
0 & 1 & 0 & 0 \\
1 & 0 & 0 & 0 \\
0 & 0 & 0 & 0 \\
0 & 0 & 0 & 0
\end{array}\right) \quad \rho\left(K_{2}\right):=i\left(\begin{array}{llll}
0 & 0 & 1 & 0 \\
0 & 0 & 0 & 0 \\
1 & 0 & 0 & 0 \\
0 & 0 & 0 & 0
\end{array}\right) \quad \rho\left(K_{3}\right):=i\left(\begin{array}{llll}
0 & 0 & 0 & 1 \\
0 & 0 & 0 & 0 \\
0 & 0 & 0 & 0 \\
1 & 0 & 0 & 0
\end{array}\right) .
\end{gathered}
$$

The variations (2.16) may be rewritten in terms of (2.17) as

$$
\delta\left(\begin{array}{c}
\tilde{\vartheta}^{0} \\
\tilde{\vartheta}^{a}
\end{array}\right)=i\left[\xi^{a} \rho\left(K_{a}\right)+\theta^{a} \rho\left(S_{a}\right)\right]\left(\begin{array}{c}
\tilde{\vartheta}^{0} \\
\tilde{\vartheta}^{a}
\end{array}\right) .
$$

Making now use of the algebraic equality

$$
\lambda^{a} \lambda^{b} \lambda^{c} \rho\left(K_{a}\right) \rho\left(K_{b}\right) \rho\left(K_{c}\right)=-|\lambda|^{2} \lambda^{a} \rho\left(K_{a}\right),
$$

we calculate

$$
\begin{aligned}
& e^{-i \lambda^{a} \rho\left(K_{a}\right)}=1-i \frac{\lambda^{a}}{|\lambda|} \rho\left(K_{a}\right) \sinh |\lambda|-\frac{\lambda^{a} \lambda^{b}}{|\lambda|^{2}} \rho\left(K_{a}\right) \rho\left(K_{b}\right)(\cosh |\lambda|-1) \\
& =\left(\begin{array}{llll}
1 & 0 & 0 & 0 \\
0 & 1 & 0 & 0 \\
0 & 0 & 1 & 0 \\
0 & 0 & 0 & 1
\end{array}\right)+\left(\begin{array}{cccc}
0 & \lambda_{1} & \lambda_{2} & \lambda_{3} \\
\lambda_{1} & 0 & 0 & 0 \\
\lambda_{2} & 0 & 0 & 0 \\
\lambda_{3} & 0 & 0 & 0
\end{array}\right) \frac{\sinh |\lambda|}{|\lambda|}+\left(\begin{array}{cccc}
|\lambda|^{2} & 0 & 0 & 0 \\
0 & \lambda_{1}^{2} & \lambda_{1} \lambda_{2} & \lambda_{1} \lambda_{3} \\
0 & \lambda_{1} \lambda_{2} & \lambda_{2}^{2} & \lambda_{2} \lambda_{3} \\
0 & \lambda_{1} \lambda_{3} & \lambda_{2} \lambda_{3} & \lambda_{3}^{2}
\end{array}\right) \frac{(\cosh |\lambda|-1)}{|\lambda|^{2}} .
\end{aligned}
$$

Thus we can rewrite (2.14) as

$$
\left(\begin{array}{c}
\vartheta^{0} \\
\vartheta^{a}
\end{array}\right)=e^{-i \lambda^{a} \rho\left(K_{a}\right)}\left(\begin{array}{c}
\tilde{\vartheta}^{0} \\
\tilde{\vartheta}^{a}
\end{array}\right)
$$


The matrix $e^{-i \lambda^{a} \rho\left(K_{a}\right)}$ performs a change of basis leading from the Lorentz covectorvalued 1 -forms in the r.h.s. of (2.21) to the $S O(3)$ quantities in the l.h.s., whose variations are specified in (2.13). In fact, taking into account the transformation properties of the coset parametre $\lambda^{a}$ as given by (2.7), it is easy to verify how the nonlinear realization splits the four-dimensional representation into the $\mathrm{SO}(3)$ singlet $\vartheta^{0}$ plus the $\mathrm{SO}(3)$ triplet $\vartheta^{a}$ respectively.

Finally, let us end this section introducing the field strengths corresponding to the gauge potentials we have defined. They will play the central role in the construction of the gauge theory of Gravitation in section 6 . In order to obtain them, let us commute two covariant differentials as defined in (A.8). We get

$$
\mathbf{D} \wedge \mathbf{D}=d \Gamma+\Gamma \wedge \Gamma=-i T^{0} P_{0}-i T^{a} P_{a}+i \mathcal{B}^{a} K_{a}+i \mathcal{R}^{a} S_{a},
$$

with the torsions

$$
\begin{aligned}
& T^{0}:=d \vartheta^{0}+\vartheta_{a} \wedge X^{a} \\
& T^{a}:=D \vartheta^{a}+\vartheta^{0} \wedge X^{a},
\end{aligned}
$$

the boost curvature

$$
\mathcal{B}^{a}:=D X^{a},
$$

and the rotational curvature

$$
\mathcal{R}^{a}:=F^{a}-\frac{1}{2} \epsilon_{b c}^{a} X^{b} \wedge X^{c},
$$

being the $S O(3)$ covariant differentials defined as

$$
\begin{aligned}
D \vartheta^{a} & :=d \vartheta^{a}+\epsilon_{b c}^{a} A^{b} \wedge \vartheta^{c} \\
D X^{a} & :=d X^{a}+\epsilon_{b c}^{a} A^{b} \wedge X^{c},
\end{aligned}
$$

and the $S O(3)$ field strength as

$$
F^{a}:=d A^{a}+\frac{1}{2} \epsilon_{b c}^{a} A^{b} \wedge A^{c} .
$$

Later we will return back to these definitions. But first we have to introduce a suitable foliation of the spacetime.

\section{Poincaré invariant foliation of spacetime}

Taking advantage of the existence of the invariant time component of the coframe, it becomes possible to perform an invariant foliation adapted to it. This will enable us to define 3-dimensional hypersurfaces in which the gravitational $S O(3)$ tensors find their natural seat. 
The general requirement for a foliation to be possible is given by Frobenius' theorem. Here we summarize briefly one of its formulations ${ }^{(15)}$. Let us depart from a 4-dimensional manifold $M$. A 3-dimensional distribution $\mathcal{D}$ on $\mathrm{M}$ is a choice of a 3-dimensional subspace $\mathcal{D}(m)$ of $M_{m}$ for each point $m$ in $M$. The distribution $\mathcal{D}$ is said to be smooth if for each $m$ in $M$ there exist a neighborhood $U$ and a basis of 3 vector fields $X_{i}$ spanning $\mathcal{D}$ at each point of $U$. A submanifold of $M$ whose tangent spaces at each point coincide with the subspaces determined by the distribution $\mathcal{D}$ is called an integral manifold of $\mathcal{D}$. Frobenius' theorem establishes that the necessary and sufficient condition for the existence of integral manifolds of $\mathcal{D}$ throw each point of $M$ (what we will call a foliation of the 4-dimensional manifold into 3-dimensional hypersurfaces) is that, whenever $X_{i}, X_{j}$ belong to $\mathcal{D}$, then the Lie derivative of these vectors with respect to each other, namely $\left[X_{i}, X_{j}\right]$, also belongs to $\mathcal{D}$. (In this case, $\mathcal{D}$ is said to be involutive or completely integrable.)

Having in mind this general result, we will perform a gauge adapted foliation which takes into account the particular coframe defined in the previous section via the nonlinear realization of the Poincaré group. From the 1-form basis (2.14) we define its dual vector basis $e_{\alpha}$ such that $\left.e_{\alpha}\right\rfloor \vartheta^{\beta}=\delta_{\alpha}^{\beta}$. In the 4-dimensional space, the Lie derivative of the basis vectors with respect to each other may be expressed in terms of the anholonomiticity objects $d \vartheta^{\alpha}$ as

$$
\left.\left.\left[e_{\alpha}, e_{\beta}\right]=\left(e_{\alpha}\right\rfloor e_{\beta}\right\rfloor d \vartheta^{\gamma}\right) e_{\gamma}
$$

Let us now separate $e_{0}$ from $e_{a}(a=1,2,3)$, since we are interested in foliating the spacetime into spacelike hypersurfaces equipped with the $S O(3)$ vector bases $e_{a}$. According to Frobenius' theorem, the necessary and sufficient condition for such a foliation to be possible is that

$$
\left.\left.\left[e_{a}, e_{b}\right]=\left(e_{a}\right\rfloor e_{b}\right\rfloor d \vartheta^{c}\right) e_{c},
$$

which is equivalent to impose the condition $\left.\left.\left(e_{a}\right\rfloor e_{b}\right\rfloor d \vartheta^{0}\right)=0$, or $\left.d \vartheta^{0}=\vartheta^{0} \wedge\left(e_{0}\right\rfloor d \vartheta^{0}\right)$. In other words,

$$
\vartheta^{0} \wedge d \vartheta^{0}=0
$$

Notice that, according to (2.13a), eq.(3.3) is Poincaré invariant. In fact, due to the ocurrence in it of an ordinary instead of a covariant differential, the condition (3.3) required by the Frobenius theorem is groupally well defined only if $\vartheta^{0}$ is invariant. Accordingly, (3.3) defines an invariant foliation. From it follows that the time coframe takes the form

$$
\vartheta^{0}=u^{0} d \tau
$$

with $\tau$ as a parametric time.

Let us now see how the dual vector $e_{0}$ of (3.4) looks like. In terms of the vector field $\partial_{\tau}$ associated to the time parametre $\tau$, we define

$$
\left.u^{\alpha}:=\partial_{\tau}\right\rfloor \vartheta^{\alpha}
$$


which represents a sort of (coordinate independent) fourvelocity due to the similarity between $\tau$ and the proper time. We can express $\partial_{\tau}$ in terms of the vector basis $e_{\alpha}$ as

$$
\partial_{\tau}=u^{\alpha} e_{\alpha}=u^{0} e_{0}+u^{a} e_{a}
$$

From (3.6) follows that the Poincaré invariant vector $e_{0}$ reads

$$
e_{0}=\frac{1}{u^{0}} \partial_{\tau}-\frac{u^{a}}{u^{0}} e_{a}
$$

We can alternatively rewrite it in terms of the three-dimensional velocity $v^{a}:=$ $c u^{a} / u^{0}$ or of the coordinate velocity $v^{A}:=e^{A}{ }_{a} v^{a}$ respectively as

$$
e_{0}=\frac{1}{u^{0}} \partial_{\tau}-\frac{v^{a}}{c} e_{a}=\frac{1}{u^{0}} \partial_{\tau}-\frac{v^{A}}{c} \partial_{A}
$$

We identify $e_{0}$ as the invariant timelike vector field along which the foliation of the spacetime is defined. Observe that the components of the fourvelocity in (3.7) relate in a simple way to the usual lapse and shift functions considered in the usual foliations of spacetime ${ }^{(12,13)}$. The main difference between them and our approach consists in that we foliate the underlying manifold taking the gauge properties of the coframe, as they arise from the nonlinear approach, as a guide. Accordingly, in the following we only will need to consider the lapse-like function $u^{0}$ explicitly, since the shift-like velocity $u^{a}$ will appear enclosed in the $S O(3)$ triads representing the space coframe, namely

$$
\vartheta^{a}=u^{a} d \tau+e_{A}^{a} d x^{A},
$$

compare with (3.4). Obviously

$$
\begin{aligned}
& \left.e_{0}\right\rfloor \vartheta^{0}=1, \\
& \left.e_{0}\right\rfloor \vartheta^{a}=0 .
\end{aligned}
$$

Any arbitrary $\mathrm{p}$-form $\alpha$ admits a decomposition into a normal and a tangential part with respect to the invariant vector field $e_{0}$ inducing the foliation, namely

$$
\alpha={ }^{\perp} \alpha+\underline{\alpha}
$$

with the normal and tangential parts respectively defined as

$$
\left.{ }^{\perp} \alpha:=\vartheta^{0} \wedge \alpha_{\perp} \quad, \quad \alpha_{\perp}:=e_{0}\right\rfloor \alpha
$$

and

$$
\left.\underline{\alpha}:=e_{0}\right\rfloor\left(\vartheta^{0} \wedge \alpha\right) .
$$

In particular we find

$$
\begin{aligned}
& \vartheta^{0}={ }^{\perp} \vartheta^{0}=u^{0} d \tau \\
& \vartheta^{a}=\underline{\vartheta}^{a}
\end{aligned}
$$


thus showing trivially that $\vartheta^{0}$ only presents a normal contribution, and $\vartheta^{a}$ only a tangential one. The general expressions for the foliation of an arbitrary $\mathrm{p}$-form $\alpha$ and its Hodge dual read respectively

$$
\begin{aligned}
\alpha & =\vartheta^{0} \wedge \alpha_{\perp}+\underline{\alpha} \\
{ }^{*} \alpha & =(-1)^{p} \vartheta^{0} \wedge{ }^{\#} \underline{\alpha}-{ }^{\#} \alpha_{\perp} .
\end{aligned}
$$

The asterisc $*$ stands for the Hodge dual in four dimensions, whereas \# represents its three-dimensional restriction, see Appendix E.

Let us define the Lie derivative with respect to $e_{0}$, namely

$$
\left.\left.l_{e_{0}} \alpha:=d\left(e_{0}\right\rfloor \alpha\right)+\left(e_{0}\right\rfloor d \alpha\right)
$$

In particular, the Lie derivative of the tangential part of a form reduces to

$$
\left.l_{e_{0}} \underline{\alpha}=\left(e_{0}\right\rfloor d \underline{\alpha}\right) .
$$

It represents the time evolution of $\underline{\alpha}$. Now we can decompose the exterior differential of an arbitrary form as

$$
d \alpha=\vartheta^{0} \wedge\left[l_{e_{0}} \underline{\alpha}-\frac{1}{u^{0}} \underline{d}\left(u^{0} \alpha_{\perp}\right)\right]+\underline{d} \underline{\alpha} .
$$

Observe that the Lie derivative of the normal part $\alpha_{\perp}$ is absent. This is a general feature of gauge theories. Finally, we have at our disposal all the elements necessary to develop a Hamiltonian approach which is well behaved in the presence of Gravity when considered as the nonlinear gauge theory of the Poincaré group.

\section{Hamiltonian formalism}

In this section we will outline the deduction of the Hamiltonian treatment of gauge theories in general, expressed in terms of differential forms. We follow essentially the work of Wallner ${ }^{(13)}$, but we make use of the invariant spacetime foliation of the previous section. The resulting formalism resembles strongly the classical one. However, being the dynamical variables differential forms, the theory is automatically diff-invariant. Furthermore, it makes it possible to take into account the gravitational effects, see sections 5,6 below.

Let us consider a general gauge theory depending on the gauge potential $A$. When we consider a particular group, $A$ will posses the corresponding group indices, but we do not need to specify them at this stage. The Lagrangian density depends on $A$ and on its exterior differential $d A$, i.e.

$$
L=L(A, d A)
$$


Since the gauge potential $A$ is a connection, it is a 1 -form which, as established in the previous section, can be decomposed into its normal and tangential parts as

$$
A=\vartheta^{0} \wedge A_{\perp}+\underline{A} .
$$

The Lagrangian density is a 4 -form. Thus, its tangential part does not exist. It decomposes simply as

$$
L=\vartheta^{0} \wedge L_{\perp} .
$$

In terms of its normal part $L_{\perp}$ we define the momenta

$$
\#^{A_{\perp}}:=\frac{\partial L_{\perp}}{\partial\left(l_{e_{0}} A_{\perp}\right)} \quad, \quad \#^{*} \pi^{\underline{A}}:=\frac{\partial L_{\perp}}{\partial\left(l_{e_{0}} \underline{A}\right)},
$$

and we construct the Hamiltonian 3-form

$$
\mathcal{H}:=u^{0}\left[l_{e_{0}} A_{\perp} \pi^{A_{\perp}}+l_{e_{0}} \underline{A} \wedge^{\#} \pi^{\underline{A}}-L_{\perp}\right] .
$$

In Appendix $\mathrm{C}$ we present a deduction of the Hamilton equations based on the comparison of the variations of (4.1) and (4.5) respectively. We do so in order to show the correctness of the results we will obtain here proceeding in a shorter and more general way, see Ref.(11). In particular, Appendix C guarantees the consistence of the definitions of the Lie derivatives used in $(4.7,8)$ below.

We begin by reconstructing the Lagrangian density in terms of the momenta and (4.5), namely

$$
L=d A_{\perp} \wedge{ }^{\#} \pi^{A_{\perp}}+d \underline{A} \wedge^{\#} \pi^{\underline{A}}-d \tau \wedge \mathcal{H},
$$

and we deduce the field equations

$$
\begin{gathered}
u^{0} l_{e_{0}} A_{\perp}:=\frac{d A_{\perp}}{d \tau}=\frac{\delta \mathcal{H}}{\delta \# \pi^{A_{\perp}}} \quad, \quad u^{0} l_{e_{0}}{ }^{\#} \pi^{A_{\perp}}:=\frac{d^{\#} \pi^{A_{\perp}}}{d \tau}=-\frac{\delta \mathcal{H}}{\delta A_{\perp}}, \\
u^{0} l_{e_{0}} \underline{A}:=\frac{d \underline{A}}{d \tau}=\frac{\delta \mathcal{H}}{\delta \# \pi^{\underline{A}}} \quad, \quad u^{0} l_{e_{0}}^{\#} \pi^{\underline{A}}:=\frac{d^{\#} \pi^{\underline{A}}}{d \tau}=-\frac{\delta \mathcal{H}}{\delta \underline{A}} .
\end{gathered}
$$

On the other hand, the Lie derivative of an arbitrary p-form defined on the 3-space and being a functional of the dynamical variables may be expanded as

$$
l_{e_{0}} \omega=l_{e_{0}} A_{\perp} \frac{\delta \omega}{\delta A_{\perp}}+l_{e_{0}} \underline{A} \wedge \frac{\delta \omega}{\delta \underline{A}}+\frac{\delta \omega}{\delta \# \pi^{A_{\perp}}} l_{e_{0}}{ }^{\#} \pi^{A_{\perp}}+\frac{\delta \omega}{\delta \# \pi^{\underline{A}}} \wedge l_{e_{0}}{ }^{\#} \pi^{\underline{A}} .
$$

We substitute the field equations $(4.7,8)$ into $(4.9)$ and accordingly we define Poisson brackets representing the time evolution of differential forms as

$$
\begin{aligned}
u^{0} l_{e_{0}} \omega=\{\omega, \mathcal{H}\}: & =\frac{\delta \mathcal{H}}{\delta \# \pi^{A_{\perp}}} \frac{\delta \omega}{\delta A_{\perp}}-\frac{\delta \omega}{\delta \# \pi^{A_{\perp}}} \frac{\delta \mathcal{H}}{\delta A_{\perp}} \\
& +\frac{\delta \mathcal{H}}{\delta^{\#} \pi^{\underline{A}}} \wedge \frac{\delta \omega}{\delta \underline{A}}-\frac{\delta \omega}{\delta \# \pi^{\underline{A}}} \wedge \frac{\delta \mathcal{H}}{\delta \underline{A}} .
\end{aligned}
$$


From definition (4.10) we obtain the basic properties of the Poisson brackets. In the first place, they are evidently antisymmetric:

$$
\{\omega, \mathcal{H}\}=-\{\mathcal{H}, \omega\}
$$

Taking into account the chain rule of the Lie derivative, namely

$$
l_{e_{0}}(\sigma \wedge \omega)=l_{e_{0}} \sigma \wedge \omega+\sigma \wedge l_{e_{0}} \omega,
$$

we get the distributive property

$$
\{\sigma \wedge \omega, \mathcal{H}\}=\{\sigma, \mathcal{H}\} \wedge \omega+\sigma \wedge\{\omega, \mathcal{H}\}
$$

Finally, since the normal part of the identity $d \wedge d \alpha \equiv 0$ reads

$$
l_{e_{0}} \underline{d} \underline{\alpha}-\frac{1}{u^{0}} \underline{d}\left(u^{0} l_{e_{0}} \underline{\alpha}\right) \equiv 0,
$$

it follows that, being $\omega$ a form defined on the 3-space and thus being absent a normal part of it, it holds

$$
\{\underline{d} \omega, \mathcal{H}\}-\underline{d}\{\omega, \mathcal{H}\}=0 .
$$

This completes the formal instrument we need to calculate the time evolution of any dynamical variable in terms of the Hamiltonian 3-form (4.5).

\section{Yang-Mills theories}

Instead of directly undertake the study of the Hamiltonian treatment of Gravitation, let us first consider the example of simpler gauge theories. This will illuminate the further developments, which are a straightforward generalization of what we will establish in this section. In particular, we will show how to deal with constraints.

Let us consider the Yang-Mills theory of an internal group with structure constants $f^{a} b c$, antisymmetric in the two last indices. Its field strength

$$
F^{a}:=d A^{a}+\frac{1}{2} f_{b c}^{a} A^{b} \wedge A^{c}
$$

decomposes into

$$
F^{a}=\vartheta^{0} \wedge F_{\perp}^{a}+\underline{F}^{a},
$$

being its normal and tangential parts respectively given by

$$
F_{\perp}^{a}:=l_{e_{0}} \underline{A}^{a}-\frac{1}{u^{0}} \underline{D}\left(u^{0} A_{\perp}^{a}\right) \quad, \quad \underline{F}^{a}:=\underline{d} \underline{A}^{a}+\frac{1}{2} f^{a} b c \underline{A}^{b} \wedge \underline{A}^{c} .
$$


According to (3.16b), its Hodge dual decomposes as

$$
{ }^{*} F^{a}=\vartheta^{0} \wedge{ }^{\#} \underline{F}^{a}-{ }^{\#} F_{\perp}^{a} .
$$

In the absence of matter, the Lagrangian density of a standard Yang-Mills theory is given by

$$
L=-\frac{1}{2} F^{a} \wedge^{*} F_{a}=\frac{1}{2} \vartheta^{0} \wedge\left(F_{\perp}^{a} \wedge{ }^{\#} F_{\perp a}-\underline{F}^{a} \wedge{ }^{\#} \underline{F}_{a}\right) .
$$

From (5.5) we get the normal part

$$
L_{\perp}=\frac{1}{2}\left(F_{\perp}^{a} \wedge{ }^{\#} F_{\perp a}-\underline{F}^{a} \wedge{ }^{\#} \underline{F}_{a}\right),
$$

and we calculate the momenta as defined in (4.4), namely

$$
\begin{gathered}
{ }^{\#} \pi_{a}^{A_{\perp}}:=\frac{\partial L_{\perp}}{\partial\left(l_{e_{0}} A_{\perp}^{a}\right)}=0, \\
{ }^{\#} \pi_{a}^{\underline{A}}:=\frac{\partial L_{\perp}}{\partial\left(l_{e_{0}} \underline{A}^{a}\right)}={ }^{\#} F_{\perp}^{a} .
\end{gathered}
$$

The first one is a primary constraint. We will come back to this fact immediately to show how to proceed in this and analogous cases. For the present, we calculate the canonical Hamiltonian 3-form (4.5) as

$$
\begin{aligned}
\mathcal{H}_{0} & :=u^{0}\left(l_{e_{0}} A_{\perp}^{a} \pi_{a}^{A_{\perp}}+l_{e_{0}} \underline{A}^{a} \wedge{ }^{\#} \pi_{a}^{\underline{A}}-L_{\perp}\right) \\
& =\frac{1}{2} u^{0}\left(\pi^{\underline{A} a} \wedge^{\#} \pi_{a}^{\underline{A}}+\underline{F}^{a} \wedge^{\#} \underline{F}_{a}\right)+\underline{D}\left(u^{0} A_{\perp}^{a}\right) \wedge^{\#} \pi_{a}^{\underline{A}} .
\end{aligned}
$$

But the time evolution operator must include information about the primary constraint (5.7). This is carried out by adding it by means of a Lagrange multiplier ${ }^{(11)}$. Doing so and integrating by parts the last term in (5.9), we get the total Hamiltonian

$$
\mathcal{H}:=\frac{1}{2} u^{0}\left(\pi^{\underline{A} a} \wedge{ }^{\#} \pi_{a}^{\underline{A}}+\underline{F}^{a} \wedge{ }^{\#} \underline{F}_{a}\right)-u^{0} A_{\perp}^{a} \underline{D}^{\#} \pi_{a}^{\underline{A}}+\beta^{a \#} \pi_{a}^{A_{\perp}} .
$$

Observe that the result is equivalent to having departed from the definition (4.5) and having replaced the Lie derivative acompanying the constraint (5.7) by a Lagrange multiplier. We retain this recipe to procede analogously in the following.

The function $u^{0}$ appearing everywhere, see (3.4), carries information about the underlying geometry and thus about the gravitational background. Nevertheless, relatively to the internal group considered here $u^{0}$ does not play the role of a dynamical 
variable. Only $A_{\perp}^{a}$ and $\underline{A}^{a}$ play this role, so that the Poisson brackets (4.10) take the form

$$
\begin{aligned}
u^{0} l_{e_{0}} \omega=\{\omega, \mathcal{H}\}: & =\frac{\delta \mathcal{H}}{\delta \# \pi_{a}^{A_{\perp}}} \frac{\delta \omega}{\delta A_{\perp}^{a}}-\frac{\delta \omega}{\delta \# \pi_{a}^{A_{\perp}}} \frac{\delta \mathcal{H}}{\delta A_{\perp}^{a}} \\
& +\frac{\delta \mathcal{H}}{\delta \# \pi_{a}^{A}} \wedge \frac{\delta \omega}{\delta \underline{A}^{a}}-\frac{\delta \omega}{\delta \# \pi_{a}^{A}} \wedge \frac{\delta \mathcal{H}}{\delta \underline{A}^{a}} .
\end{aligned}
$$

As before, see $(4.11,13)$, we have

$$
\{\omega, \mathcal{H}\}=-\{\mathcal{H}, \omega\}
$$

and

$$
\{\sigma \wedge \omega, \mathcal{H}\}=\{\sigma, \mathcal{H}\} \wedge \omega+\sigma \wedge\{\omega, \mathcal{H}\}
$$

but in addition to (4.15), which remains valid, we can now generalize it in order to take into account the contributions due to the connections appearing in the covariant differentials. Departing from the identity

$$
u^{0} \mathrm{E}_{e_{0}} \underline{D} \omega^{a}-\underline{D}\left(u^{0} \mathrm{E}_{e_{0}} \omega^{a}\right) \equiv u^{0} f_{b c}^{a} F_{\perp}^{b} \wedge \omega^{c},
$$

being $\omega^{a}$ defined on the three-space, we get

$$
\left\{\underline{D} \omega^{a}, \mathcal{H}\right\}-\underline{D}\left\{\omega^{a}, \mathcal{H}\right\}=f_{b c}^{a}\left\{\underline{A}^{b}, \mathcal{H}\right\} \wedge \omega^{c}
$$

Now we require the stability of the constraint (5.7), i.e. we impose that its time derivative vanishes. In terms of the Poisson brackets we calculate its time evolution

$$
u^{0} l_{e_{0}}^{\#} \pi_{a}^{A_{\perp}}=\left\{{ }^{\#} \pi_{a}^{A_{\perp}}, \mathcal{H}\right\}=u^{0} \underline{D}^{\#} \pi_{a}^{\underline{A}},
$$

and we put it equal to zero. The resulting stability condition

$$
\underline{D}^{\#} \pi_{a}^{\underline{A}}=0
$$

is a secondary constraint. Further we require the time stability of (5.17). Making use of $(5.15)$, we find

$$
u^{0} l_{e_{0}} \underline{D}^{\#} \pi_{a}^{\underline{A}}=\left\{\underline{D}^{\#} \pi_{a}^{\underline{A}}, \mathcal{H}\right\}=-u^{0} f_{a b}^{c} A_{\perp}^{b} \underline{D}^{\#} \pi_{c}^{\underline{A}}+2 f_{(a b)}{ }^{c} \underline{D}\left(u^{0} A_{\perp}^{b} \pi_{c}^{\underline{A}}\right) .
$$

Assuming that we are dealing with a group whose structure constants are antisymmetric in the first indices, eq.(5.18) reduces to

$$
\mathrm{E}_{e_{0}} \underline{D}^{\#} \pi_{a}^{\underline{A}}=0
$$


Thus, the constraint (5.17) is automatically stable and the search for constraints is finished. The evolution of the system takes place in a submanifold of the phase space defined by (5.7) and (5.17). The evolution equations are easily obtained as

$$
\begin{gathered}
u^{0} l_{e_{0}} \underline{A}^{a}=\left\{\underline{A}^{a}, \mathcal{H}\right\}=u^{0} \pi^{\underline{A} a}+\underline{D}\left(u^{0} A_{\perp}^{a}\right), \\
u^{0} l_{e_{0}}^{\#} \pi_{a}^{\underline{A}}=\left\{\# \pi_{a}^{\underline{A}}, \mathcal{H}\right\}=-\underline{D}\left(u^{0 \#} \underline{F}_{a}\right)-u^{0} \bar{\eta}_{a b}^{c} A_{\perp}^{b} \pi_{c}^{\underline{A}} .
\end{gathered}
$$

We rewrite $(5.20,21)$ in the explicitly covariant form

$$
\begin{gathered}
\pi^{\underline{A} a}=l_{e_{0}} \underline{A}^{a}-\frac{1}{u^{0}} \underline{D}\left(u^{0} A_{\perp}^{a}\right)=: F_{\perp}^{a}, \\
\mathrm{E}_{e_{0}}{ }^{\#} \pi_{a}^{\underline{A}}+\frac{1}{u^{0}} \underline{D}\left(u^{0} \underline{F}_{a}\right)=0 .
\end{gathered}
$$

In addition, the remaining equation

$$
u^{0} l_{e_{0}} A_{\perp}^{a}=\left\{A_{\perp}^{a}, \mathcal{H}\right\}=\beta^{a}
$$

fixes the value of the Lagrange multiplier.

Let us now compare our Hamiltonian equations with the usual four-dimensional Lagrangian equations $D^{*} F^{a}=0$. They decompose into their normal and tangential parts as

$$
D^{*} F^{a}=-\vartheta^{0} \wedge\left[\mathrm{E}_{e_{0}}{ }^{\#} F_{\perp}^{a}+\frac{1}{u^{0}} \underline{D}\left(u^{0} \underline{F}^{a}\right)\right]-\underline{D}^{\#} F_{\perp}^{a} .
$$

Since according to $(5.22)$ the momentum $\pi^{\underline{A} a}$ coincides with $F_{\perp}^{a}$, we see that the evolution equation (5.23) corresponds to the normal part of (5.25), and the constraint (5.17) to the tangential part.

\section{Hamiltonian treatment of Gravitation}

At last we arrive at the main application of the general theory established in sections 2-4. The nonlinear realization of the Poincaré group and the Poincaré invariant foliation of spacetime naturally related to it constitute the foundations of the Hamiltonian approach to Gravity. In this context, we could of course choose as our starting point to derive the dynamical equations a very general action including quadratic terms. But our purpose in this paper is to show how our formalism works, and the best way to do so is to depart from the Einstein-Cartan action, which gives rise to the standard Einstein equations of GR. In fact, we will be able to derive them, see (7.8-14,18) below, thus showing that our approach comprises the usual Lagrangian one at the level of the field equations. However, our treatment is in some extent more restrictive, see section 8 below, and it has the additional virtue of distinguishing 
between those parts of the Einstein equations which are true evolution equations and those which correspond to constraints on the phase space. Thus we depart from the Einstein-Cartan Lagrange density with cosmological constant

$$
L=-\frac{1}{2 l^{2}} R_{\alpha}^{\beta} \wedge \eta_{\beta}^{\alpha}+\frac{\Lambda}{l^{2}} \eta
$$

Let us reexpress the Einstein-Cartan term

$$
-\frac{1}{2 l^{2}} R_{\alpha}^{\beta} \wedge \eta_{\beta}^{\alpha}=-\frac{1}{2 l^{2}}\left(2 R^{0 a} \wedge \eta_{0 a}+R^{a b} \wedge \eta_{a b}\right)
$$

in terms of the threedimensional dynamical variables defined in section 2 . We point out once more that in our approach the interactions are not mediated by the metric, which appears in the theory as a trivial, nondynamical, anholonomic Kronecker delta, but by the (nonlinear) connections.

According to (2.12), the components of the fourdimensional nonlinear Lorentz connections relate to the corresponding $S O(3)$ quantities as

$$
\Gamma^{0 a}=X^{a}, \quad \Gamma^{a b}=\epsilon_{c}^{a b} A^{c}
$$

and accordingly, see $(2.22,24,25)$,

$$
R^{0 a}=D X^{a} \quad, \quad R^{a b}=\epsilon^{a b}{ }_{c} \mathcal{R}^{c} .
$$

On the other hand, from (E.1) follows

$$
\eta_{0 a}=\bar{\eta}_{a} \quad, \quad \eta_{a b}=\vartheta^{0} \wedge \bar{\eta}_{a b}
$$

Thus, substituting $(6.4,5)$ into $(6.2)$ and performing at the same time the decomposition into the normal and tangential parts of the quantities involved as established in section 3 , we get

$$
-\frac{1}{2 l^{2}} R_{\alpha}{ }^{\beta} \wedge \eta_{\beta}^{\alpha}=-\frac{1}{l^{2}} \vartheta^{0} \wedge\left\{\left[\mathrm{E}_{e_{0}} \underline{X}^{a}-\frac{1}{u^{0}} \underline{D}\left(u^{0} X_{\perp}^{a}\right)\right] \wedge \bar{\eta}_{a}+\frac{1}{2} \epsilon^{a b}{ }_{c} \underline{\mathcal{R}}^{c} \wedge \bar{\eta}_{a b}\right\}
$$

where we have made use of the definitions of the covariant Lie derivative and the covariant tangential differential respectively, namely

$$
\begin{aligned}
\mathrm{Ł}_{e_{0}} \underline{X}^{a} & \left.\left.:=e_{0}\right\rfloor D \underline{X}^{a}=e_{0}\right\rfloor\left(d \underline{X}^{a}+\epsilon_{b c}^{a} A^{b} \wedge \underline{X}^{c}\right) \\
\underline{D}\left(u^{0} X_{\perp}^{a}\right) & :=\underline{d}\left(u^{0} X_{\perp}^{a}\right)+\epsilon_{b c}^{a} \underline{A}^{b} u^{0} X_{\perp}^{c} .
\end{aligned}
$$

According to $(2.25,27)$, the tangential part of the curvature present in (6.6) reads

$$
\underline{\mathcal{R}}^{a}:=\underline{F}^{a}-\frac{1}{2} \epsilon_{b c}^{a} \underline{X}^{b} \wedge \underline{X}^{c} \quad, \quad \underline{F}^{a}:=\underline{d} \underline{A}^{a}+\frac{1}{2} \epsilon_{b c}^{a} \underline{A}^{b} \wedge \underline{A}^{c} .
$$


For later convenience we also give here the structure of the normal part of the curvature

$$
\mathcal{R}_{\perp}^{a}:=F_{\perp}^{a}-\epsilon_{b c}^{a} X_{\perp}^{b} \underline{X}^{c} \quad, \quad F_{\perp}^{a}:=l_{e_{0}} \underline{A}^{a}-\frac{1}{u^{0}} \underline{D}\left(u^{0} A_{\perp}^{a}\right) .
$$

Despite it does not appear in the action, it will be present in the field equations.

Due to the fact that the holonomic $S O(3)$ metric is the Kronecker delta, we have $\bar{\eta}_{a b c}=\#\left(\vartheta_{a} \wedge \vartheta_{b} \wedge \vartheta_{c}\right)=\sqrt{\operatorname{det}\left(\delta_{m n}\right)} \epsilon_{a b c}=\epsilon_{a b c}$, see (E.1). Thus we identify this object with the group constants of $S O(3)$ in order to simplify the expression of the last term in (6.6). After integrating by parts to make explicit the dependence on $\mathrm{Ł}_{e_{0}} \vartheta^{a}$, we get the normal part of the Lagrangian

$$
L_{\perp}=-\frac{1}{l^{2}}\left\{\frac{1}{2}\left[\mathrm{E}_{e_{0}} \underline{X}^{a} \wedge \bar{\eta}_{a}-\underline{X}^{a} \wedge \mathrm{E}_{e_{0}} \bar{\eta}_{a}\right]-\frac{1}{u^{0}} \underline{D}\left(u^{0} X_{\perp}^{a}\right) \wedge \bar{\eta}_{a}+\vartheta^{a} \wedge \underline{\mathcal{R}}_{a}-\Lambda \bar{\eta}\right\} .
$$

Now we follow the steps of section 4 , taking into account the results of section 5 concerning the treatment of constrained systems. First we define the momenta

$$
\begin{aligned}
\# \pi^{u^{0}}: & :=\frac{\partial L_{\perp}}{\partial\left(l_{e_{0}} u^{0}\right)}=0 \\
\# \pi_{a}^{\vartheta} & :=\frac{\partial L_{\perp}}{\partial\left(l_{e_{0}} \vartheta^{a}\right)}=-\frac{1}{2 l^{2}} \bar{\eta}_{a b} \wedge \underline{X}^{b} \\
\# \pi_{a}^{A_{\perp}}: & :=\frac{\partial L_{\perp}}{\partial\left(l_{e_{0}} A_{\perp}^{a}\right)}=0 \\
{ }^{\#} \pi_{a}^{\underline{A}} & :=\frac{\partial L_{\perp}}{\partial\left(l_{e_{0}} \underline{A}^{a}\right)}=0 \\
{ }^{*} \pi_{a}^{X_{\perp}}: & :=\frac{\partial L_{\perp}}{\partial\left(l_{e_{0}} X_{\perp}^{a}\right)}=0 \\
\# \pi_{a}^{\underline{x}} & :=\frac{\partial L_{\perp}}{\partial\left(l_{e_{0}} \underline{X}^{a}\right)}=-\frac{1}{2 l^{2}} \bar{\eta}_{a} .
\end{aligned}
$$

Observe that all of them are constraints. These are the primary constraints which have to be included in the time evolution operator by means of Lagrange multipliers, as in (5.10). It is interesting to notice that, acording to the last equation in (6.11), the momenta conjugated to $\underline{X}^{a}$ are proportional to the triads, i.e. $\pi_{a}^{\underline{X}}=-\frac{1}{2 l^{2}} \vartheta_{a}$. The canonical Hamiltonian, see (5.9), reads

$$
\begin{aligned}
\mathcal{H}_{0}:=u^{0}( & l_{e_{0}} u^{0} \pi^{u^{0}}+l_{e_{0}} \vartheta^{a} \wedge{ }^{\#} \pi_{a}^{\vartheta}+l_{e_{0}} A_{\perp}^{a} \pi_{a}^{A_{\perp}} \\
& \left.+l_{e_{0}} \underline{A}^{a} \wedge{ }^{\#} \pi_{a}^{\underline{A}}+l_{e_{0}} X_{\perp}^{a} \pi_{a}^{X_{\perp}}+l_{e_{0}} \underline{X}^{a} \wedge{ }^{\#} \pi_{a}^{\underline{x}}-L_{\perp}\right) .
\end{aligned}
$$

In order to construct the total Hamiltonian playing the role of the time evolution operator, we apply the recipe established in section 5. We put (6.12) into a form 
analogous to (5.10) in the following sense. By adding and substracting suitable terms, we rewrite (6.12), whenever possible, in terms of covariant expressions. In this way, the remaining noncovariant contributions are rearranged into the terms multiplied by $A_{\perp}^{a}$ and $l_{e_{0}} A_{\perp}^{a}$ respectively. Then we substitute the factors multiplying the primary constraints (6.11) by Lagrange multipliers $\beta^{i}$, as in (5.10). (All the substituted terms depend on time Lie derivatives, see the comment immediately after (5.10).) The resulting total Hamiltonian 3-form reads

$$
\begin{aligned}
\mathcal{H}=u^{0}\{ & \frac{1}{l^{2}}\left(X_{\perp}^{a} \underline{D} \bar{\eta}_{a}+\vartheta_{a} \wedge \underline{\mathcal{R}}^{a}-\Lambda \bar{\eta}\right) \\
- & \left.A_{\perp}^{a}\left[\underline{D}^{\#} \pi_{a}^{\underline{A}}+\bar{\eta}_{a b}^{c}\left(X_{\perp}^{b \#} \pi_{c}^{X_{\perp}}+\underline{X}^{b} \wedge{ }^{\#} \pi_{c}^{\underline{x}}+\vartheta^{b} \wedge \# \pi_{c}^{\vartheta}\right)\right]\right\} \\
& +\beta^{0 \#} \pi^{u^{0}}+\beta_{1}^{a} \wedge\left({ }^{\#} \pi_{a}^{\vartheta}+\frac{1}{2 l^{2}} \bar{\eta}_{a b} \wedge \underline{X}^{b}\right)+\beta_{2}^{a \#} \pi_{a}^{A_{\perp}} \\
& +\beta_{3}^{a} \wedge^{\#} \pi_{a}^{\underline{A}}+\beta_{4}^{a} \# \pi_{a}^{X_{\perp}}+\beta_{5}^{a} \wedge\left({ }^{\#} \pi_{a}^{\underline{x}}+\frac{1}{2 l^{2}} \bar{\eta}_{a}\right) .
\end{aligned}
$$

The time evolution of any dynamical variable is calculable with the help of the Poisson brackets, which take the general form

$$
\begin{aligned}
u^{0} l_{e_{0}} \omega=\{\omega, \mathcal{H}\}: & =\frac{\delta \mathcal{H}}{\delta \# \pi^{u^{0}}} \frac{\delta \omega}{\delta u^{0}}-\frac{\delta \omega}{\delta \# \pi^{u^{0}}} \frac{\delta \mathcal{H}}{\delta u^{0}} \\
& +\frac{\delta \mathcal{H}}{\delta \# \pi_{a}^{\vartheta}} \wedge \frac{\delta \omega}{\delta \vartheta^{a}}-\frac{\delta \omega}{\delta \# \pi_{a}^{\vartheta}} \wedge \frac{\delta \mathcal{H}}{\delta \vartheta^{a}} \\
& +\frac{\delta \mathcal{H}}{\delta \# \pi_{a}^{A_{\perp}}} \frac{\delta \omega}{\delta A_{\perp}^{a}}-\frac{\delta \omega}{\delta \# \pi_{a}^{A_{\perp}}} \frac{\delta \mathcal{H}}{\delta A_{\perp}^{a}} \\
& +\frac{\delta \mathcal{H}}{\delta \# \pi_{a}^{A}} \wedge \frac{\delta \omega}{\delta \underline{A^{a}}}-\frac{\delta \omega}{\delta \# \pi_{a}^{A}} \wedge \frac{\delta \mathcal{H}}{\delta \underline{A}^{a}} \\
& +\frac{\delta \mathcal{H}}{\delta \# \pi_{a}^{X}} \frac{\delta \omega}{\delta X_{\perp}^{a}}-\frac{\delta \omega}{\delta \# \pi_{a}^{X}} \frac{\delta \mathcal{H}}{\delta X_{\perp}^{a}} \\
& +\frac{\delta \mathcal{H}}{\delta \# \pi_{a}^{\underline{X}}} \wedge \frac{\delta \omega}{\delta \underline{X}^{a}}-\frac{\delta \omega}{\delta \# \pi_{a}^{x}} \wedge \frac{\delta \mathcal{H}}{\delta \underline{X}^{a}}
\end{aligned}
$$

They posses analogous properties to (5.11), namely

$$
\begin{gathered}
\{\omega, \mathcal{H}\}=-\{\mathcal{H}, \omega\}, \\
\{\sigma \wedge \omega, \mathcal{H}\}=\{\sigma, \mathcal{H}\} \wedge \omega+\sigma \wedge\{\omega, \mathcal{H}\},
\end{gathered}
$$

and

$$
\left\{\underline{D} \omega^{a}, \mathcal{H}\right\}-\underline{D}\left\{\omega^{a}, \mathcal{H}\right\}=\bar{\eta}^{a}{ }_{b c}\left\{\underline{A}^{b}, \mathcal{H}\right\} \wedge \omega^{c}
$$

compare with $(5.12,13,15)$. 
Making use of (6.14), we calculate the evolution equations of the primary constraints (6.11). The stability requirement gives rise to four secondary constraints and to conditions on two Lagrange multipliers respectively. In fact, we get the equations

$$
\begin{aligned}
u^{0} l_{e_{0}}^{\#} \pi^{u^{0}} & =-\frac{1}{l^{2}}\left(\varphi^{(0)}+X_{\perp}^{a} \varphi_{a}^{(3)}\right)+A_{\perp}^{a} \varphi_{a}^{(1)} \\
u^{0} l_{e_{0}}^{\#} \pi_{a}^{A_{\perp}} & =u^{0} \varphi_{a}^{(1)} \\
u^{0} \mathrm{E}_{e_{0}}^{\#} \pi_{a}^{\underline{A}} & =-\frac{1}{l^{2}} \varphi_{a}^{(2)} \\
u^{0} \mathrm{E}_{e_{0}}^{\#} \pi_{a}^{X_{\perp}} & =-\frac{u^{0}}{l^{2}} \varphi_{a}^{(3)} \\
u^{0} \mathrm{E}_{e_{0}}\left(\# \pi_{a}^{\underline{X}}+\frac{1}{2 l^{2}} \bar{\eta}_{a}\right) & =-\frac{1}{l^{2}} \bar{\eta}_{a b} \wedge\left(\beta_{1}^{b}+u^{0} \underline{X}^{b}\right) \\
u^{0} \mathrm{E}_{e_{0}}\left(\# \pi_{a}^{\vartheta}+\frac{1}{2 l^{2}} \bar{\eta}_{a b} \wedge \underline{X}^{b}\right) & =-\frac{1}{l^{2}}\left\{\left[\beta_{5}^{b}-\underline{D}\left(u^{0} X_{\perp}^{b}\right)\right] \wedge \bar{\eta}_{a b}+u^{0}\left(\underline{\mathcal{R}}_{a}-\Lambda \bar{\eta}_{a}\right)\right\}
\end{aligned}
$$

whose r.h.s.'s must vanish. The new secondary constraints in (6.18) are defined as

$$
\begin{aligned}
\varphi^{(0)} & :=\vartheta_{a} \wedge \underline{\mathcal{R}}^{a}-\Lambda \bar{\eta} \\
\varphi_{a}^{(1)} & :=\underline{D}^{\#} \pi_{a}^{\underline{A}}+\bar{\eta}_{a b}^{c}\left(X_{\perp}^{b} \pi_{c}^{X_{\perp}}+\underline{X}^{b} \wedge \pi_{c}^{\underline{X}}+\vartheta^{b} \wedge \# \pi_{c}^{\vartheta}\right) \\
\varphi_{a}^{(2)} & :=\underline{D}\left(u^{0} \vartheta_{a}\right)+u^{0} X_{\perp}^{b} \vartheta_{b} \wedge \vartheta_{a} \\
\varphi_{a}^{(3)} & :=\underline{D} \bar{\eta}_{a} .
\end{aligned}
$$

In addition, from $(6.18 \mathrm{e}, \mathrm{f})$ also follows that the Lagrange multipliers $\beta_{1}^{a}$ and $\beta_{5}^{a}$ satisfy respectively the equations

$$
\beta_{1}^{a}=-u^{0} \underline{X}^{a}
$$

and

$$
\frac{1}{u^{0}}\left[\beta_{5}^{b}-\underline{D}\left(u^{0} X_{\perp}^{b}\right)\right] \wedge \bar{\eta}_{a b}+\underline{\mathcal{R}}_{a}-\Lambda \bar{\eta}_{a}=0
$$

From the latter we find

$$
\left.\left.\beta_{5}^{a}=\underline{D}\left(u^{0} X_{\perp}^{a}\right)+u^{0}\left[\bar{\eta}^{a b c}\left(e_{b}\right\rfloor \underline{\mathcal{R}}_{c}\right)+\frac{1}{2} \vartheta^{a}\left(e_{b}\right\rfloor^{\#} \underline{\mathcal{R}}^{b}\right)+\frac{\Lambda}{2} \vartheta^{a}\right]
$$

Let us now examine the consequences of the secondary constraints (6.19). In view of the primary constraints $(6.11), \varphi_{a}^{(1)}$ reduces weakly to $\varphi_{a}^{(1)}=-\frac{1}{l^{2}} \vartheta_{a} \wedge \vartheta_{b} \wedge \underline{X}^{b}$. On the other hand, the constraints $\varphi_{a}^{(2)}$ and $\varphi_{a}^{(3)}$ are not independent from each other. In fact, the vanishing of $\varphi_{a}^{(3)}$ implies $\underline{D} \bar{\eta}_{a}=\underline{D} \vartheta^{b} \wedge \bar{\eta}_{a b}=0$. Substituting $\underline{D} \vartheta^{b}=$ $-\left(\underline{d} \log u^{0}+X_{\perp}^{a} \vartheta_{a}\right) \wedge \vartheta^{b}$ as deduced from $\varphi_{a}^{(2)}$, we get $\left.\#\left(e_{a}\right\rfloor \underline{d} \log u^{0}+X_{\perp}^{b} \vartheta_{b}\right)=0$, 
thus proving that $\underline{d} \log u^{0}+X_{\perp}^{a} \vartheta_{a}$ is a constraint by itself. Thus, $\underline{D} \vartheta^{a}$ also vanishes. The latter condition suffices to deduce $\varphi_{a}^{(3)}$. We conclude that the new conditions imposed by the secondary constraints (6.19) on the phase space manifold are

$$
\begin{aligned}
\vartheta_{a} \wedge \underline{\mathcal{R}}^{a}-\Lambda \bar{\eta} & =0 \\
\vartheta_{a} \wedge \underline{X}^{a} & =0 \\
\underline{d} \log u^{0}+X_{\perp}^{a} \vartheta_{a} & =0 \\
\underline{D} \vartheta^{a} & =0 .
\end{aligned}
$$

Let us now require the stability of the secondary constraints (6.19). Their time evolution calculated from the Hamiltonian (6.13) depends on contributions which are weakly equal to zero in view of (6.11) and (6.19) themselves -or equivalently (6.23)-, plus additional terms which have to vanish in order to guarantee the stability. Firstly we verify that the stability of $\varphi_{a}^{(1)}$ is automatically fulfilled since

$$
u^{0} \mathrm{E}_{e_{0}} \varphi_{a}^{(1)} \approx 0
$$

(The $\approx$ term indicates that the equation holds weakly.) New conditions on the Lagrange multipliers are obtained when considering the stability of $\varphi_{a}^{(2)}$ and $\varphi_{a}^{(3)}$ in (6.19). We find

$$
u^{0} \mathrm{E}_{e_{0}} \varphi_{a}^{(2)} \approx-u^{0}\left(\bar{\eta}_{a b} \wedge \beta_{3}^{b}-\underline{D} \beta_{a}^{1}\right)-u^{0} \vartheta_{a} \wedge\left[\underline{d}\left(\frac{\beta^{0}}{u^{0}}\right)+\beta_{4}^{b} \vartheta_{b}+X_{\perp}^{b} \beta_{b}^{1}\right]
$$

and

$$
u^{0} \mathrm{E}_{e_{0}} \varphi_{a}^{(3)}=-\left[\beta_{3}^{b} \wedge \vartheta_{b} \wedge \vartheta_{a}+\underline{D}\left(\bar{\eta}_{a b} \wedge \beta_{1}^{b}\right)\right] .
$$

Making use of the value of $\beta_{1}^{a}$ as given by $(6.20)$, it follows from $(6.25,26)$ on the one hand

$$
\underline{d}\left(\frac{\beta^{0}}{u^{0}}\right)+\beta_{4}^{a} \vartheta_{a}-u^{0} X_{\perp}^{a} \underline{X}_{a}=0
$$

which guarantees that $\mathrm{E}_{e_{0}}\left(\underline{d} \log u^{0}+X_{\perp}^{a} \vartheta_{a}\right)=0$, in view of the meaning of $\beta^{0}$ and $\beta_{4}^{a}$ deduced in (6.32) below. On the other hand

$$
\bar{\eta}_{a b} \wedge \beta_{3}^{b}+\underline{D}\left(u^{0} \underline{X}_{a}\right)=0
$$

from which we get

$$
\left.\left.\beta_{3}^{a}=-\bar{\eta}^{a b c}\left[e_{b}\right\rfloor \underline{D}\left(u^{0} \underline{X}_{c}\right)\right]-\frac{1}{2} \vartheta^{a}\left\{e_{b}\right\rfloor^{\#}\left[\underline{D}\left(u^{0} \underline{X}^{b}\right)\right]\right\} .
$$


Finally we impose the stability condition on $\varphi^{(0)}$, see (6.19a). Taking into account the equations $(6.20,21,28)$ satisfied by $\beta_{1}^{a}, \beta_{3}^{a}$ and $\beta_{5}^{a}$, we calculate

$$
u^{0} l_{e_{0}} \varphi^{(0)} \approx-\underline{d} u^{0} \wedge \varphi^{(4)}-\underline{d}\left(u^{0} \varphi^{(4)}\right),
$$

with the new constraint $\varphi^{(4)}$ defined as

$$
\varphi^{(4)}:=\vartheta_{a} \wedge{ }^{\#} \underline{D} \underline{X}^{a}
$$

This constraint is stable. Thus, our search for the constraints of the theory is finished.

We end this section giving the evolution equations of the canonical coordinates of the theory. They read

$$
\begin{aligned}
u^{0} l_{e_{0}} u^{0} & =\beta^{0} \\
u^{0} \mathrm{E}_{e_{0}} \vartheta^{a} & =\beta_{1}^{a} \\
u^{0} l_{e_{0}} A_{\perp}^{a} & =\beta_{2}^{a} \\
u^{0} F_{\perp}^{a} & =\beta_{3}^{a} \\
u^{0} \mathrm{E}_{e_{0}} X_{\perp}^{a} & =\beta_{4}^{a} \\
u^{0} \mathrm{E}_{e_{0}} \underline{X}^{a} & =\beta_{5}^{a} .
\end{aligned}
$$

(In (6.32d) we made use of definition (6.9).) The meaning of (6.32) will become clear below in view of the conditions established previously for the Lagrange multipliers. We do not write down the evolution equations of the canonical momenta (6.11) since most of them are zero constraints whose evolution equations have already been studied above, and those of the nonvanishing momenta $\# \pi_{a}^{\vartheta}$ and $\# \pi_{a}^{\underline{x}}$ are redundant with (6.32) due to the constraints (6.11) themselves.

\section{Comparison to the standard Einstein theory}

At the end of section 5, we compared the Hamiltonian equations of a Yang-Mills theory with the usual fourdimensional Lagrangian equations derived from the same action and we saw that they coincide. Here we will do the same with the gravitational equations. The fourdimensional (Lagrangian) version of them, obtained varying with respect to $\Gamma_{\alpha}{ }^{\beta}$ and $\vartheta^{\alpha}$ respectively, reads

$$
D \eta_{\alpha \beta}=0
$$

and

$$
\frac{1}{2} \eta_{\alpha \beta \gamma} \wedge R^{\beta \gamma}-\Lambda \eta_{\alpha}=0
$$


The curvature $R_{\alpha}{ }^{\beta}$ in (7.2) as much as the covariant differential in (7.1) are defined in terms of the fourdimensional Lorentz connection $\Gamma_{\alpha}{ }^{\beta}$. Eq.(7.1) establishes the vanishing of the torsion. Thus we will substitute it by

$$
T^{\alpha}=0 .
$$

As a consequence of (7.3), the Lorentz connection reduces to the Christoffel symbol ${ }^{(6,7)}$

$$
\left.\left.\left.\Gamma_{\alpha \beta}^{\{\}}:=e_{[\alpha}\right\rfloor d \vartheta_{\beta]}-\frac{1}{2}\left(e_{\alpha}\right\rfloor e_{\beta}\right\rfloor d \vartheta^{\gamma}\right) \vartheta_{\gamma},
$$

and (7.2) coincides with the standard Einstein vacuum equations with cosmological constant defined on a Riemannian space.

Let us now decompose the time and space components of the Lagrangian equations into their normal and tangential parts respectively, according to the foliation procedure of section 3. For the torsion equation (7.3) we find, see (2.23)

$$
\begin{aligned}
& 0=T^{0}:=d \vartheta^{0}+\vartheta_{a} \wedge X^{a}=-\vartheta^{0} \wedge\left(\underline{d} \log u^{0}+X_{\perp}^{a} \vartheta_{a}\right)+\vartheta_{a} \wedge \underline{X}^{a} \\
& 0=T^{a}:=D \vartheta^{a}+\vartheta^{0} \wedge X^{a}=\vartheta^{0} \wedge\left(\mathrm{E}_{e_{0}} \vartheta^{a}+\underline{X}^{a}\right)+\underline{D} \vartheta^{a} .
\end{aligned}
$$

On the other hand, the Einstein equations (7.2) decompose as

$$
0=\frac{1}{2} \eta_{0 \beta \gamma} \wedge R^{\beta \gamma}-\Lambda \eta_{0}=-\vartheta^{0} \wedge\left(\vartheta_{a} \wedge \mathcal{R}_{\perp}^{a}\right)+\left(\vartheta_{a} \wedge \underline{\mathcal{R}}^{a}-\Lambda \bar{\eta}\right)
$$

and

$$
\begin{aligned}
0 & =\frac{1}{2} \eta_{a \beta \gamma} \wedge R^{\beta \gamma}-\Lambda \eta_{a} \\
& =-\vartheta^{0} \wedge\left\{\left[\mathrm{E}_{e_{0}} \underline{X}^{b}-\frac{1}{u^{0}} \underline{D}\left(u^{0} X_{\perp}^{b}\right)\right] \wedge \bar{\eta}_{a b}+\underline{\mathcal{R}}_{a}-\Lambda \bar{\eta}_{a}\right\}-\bar{\eta}_{a b} \wedge \underline{D}^{b} .
\end{aligned}
$$

Our task now is to compare the set of Lagrangian equations (7.5-7) with our Hamiltonian ones. In order to do it, we will rearrange the results of section 6 , in particular equations $(6.20,21,28)$ cum $(6.32)$, into more explicit expressions, see below. As a general result, the tangential parts of all the Lagrangian equations vanish due to the secondary constraints we have found. In fact, the dynamical meaning of the constraints becomes transparent when comparing with (7.5-7). We read out from (6.23) the following conditions. For $T^{0}$, compare $(6.23 \mathrm{~b})$ and $(7.5 \mathrm{a})$ :

$$
\vartheta_{a} \wedge \underline{X}^{a}=0
$$

for $T^{a}$, compare $(6.23 \mathrm{~d})$ and $(7.5 \mathrm{~b})$ :

$$
\underline{D} \vartheta^{a}=0
$$


and for the time component of the Einstein equations, compare (6.23a) and (7.6):

$$
\vartheta_{a} \wedge \underline{\mathcal{R}}^{a}-\Lambda \bar{\eta}=0
$$

Furthermore, from the constraint (6.31) follows immediately the wanishing of the tangential part of the space components of the Einstein equations (7.7), namely

$$
\bar{\eta}_{a b} \wedge \underline{D}^{b}=0
$$

In short, the constraints $(7.8-11)$ are the tangential parts of the Lagrange equations (7.5-7). Sequently let us pay attention to the normal parts. With the only exception of that of $T^{0}$, see $(7.5 \mathrm{a})$, which appears as the constraint $(6.23 \mathrm{c})$ :

$$
\underline{d} \log u^{0}+X_{\perp}^{a} \vartheta_{a}=0
$$

the normal parts are obtained from the evolution equations (6.32) and the conditions $(6.20,21,28)$ on the Lagrange multipliers. Putting together $(6.32 \mathrm{~b})$ and $(6.20)$, it follows

$$
\mathrm{E}_{e_{0}} \vartheta^{a}+\underline{X}^{a}=0
$$

compare with the normal part of $(7.5 \mathrm{~b})$. On the other hand, substituting (6.32f) into (6.21), we get

$$
\left[\mathrm{E}_{e_{0}} \underline{X}^{b}-\frac{1}{u^{0}} \underline{D}\left(u^{0} X_{\perp}^{b}\right)\right] \wedge \bar{\eta}_{a b}+\underline{\mathcal{R}}_{a}-\Lambda \bar{\eta}_{a}=0
$$

which corresponds to the normal part of (7.7). Finally, from (6.28) and (6.32d) we find

$$
u^{0} \bar{\eta}_{a b} \wedge F_{\perp}^{b}+\underline{D}\left(u^{0} \underline{X}_{a}\right)=0,
$$

which, after an obvious manipulation, gives rise to

$$
\vartheta_{a} \wedge \vartheta_{b} \wedge F_{\perp}^{b}+\underline{d} \log u^{0} \wedge \bar{\eta}_{a b} \wedge \underline{X}^{b}-\bar{\eta}_{a b} \wedge \underline{D}_{\underline{X}}^{b}=0
$$

Taking into account $(7.11,12)$ and the fact that (7.8) implies $\left.e_{[a}\right\rfloor \underline{X}_{b]}=0$, the contraction of (7.16) with $e_{a}$ leads to

$$
\vartheta_{a} \wedge\left(F_{\perp}^{a}-\bar{\eta}_{b c}^{a} X_{\perp}^{b} \underline{X}^{c}\right)=0
$$

According to definition (6.9), the previous equation may be rewritten as

$$
\vartheta_{a} \wedge \mathcal{R}_{\perp}^{a}=0
$$

which reproduces the normal part of (7.6). Thus, we were able to reproduce all the Einstein equations from our Hamiltonian approach. 


\section{The $\mathbf{S O}(3)$ formulation of Einstein's equations}

Once we have proven that the usual Lagrangian equations are a consequence of our treatment, let us write down the Hamiltonian evolution equations in a more suitable and simple form, which shows more clearly their physical meaning. As a consequence, we will see that our equations (8.7-10) below are more restrictive than those of the Einstein theory. In the first place, we reexpress (7.12) as

$$
\left.X_{\perp}^{a}=-\left(e^{a}\right\rfloor \underline{d} \log u^{0}\right) .
$$

From (7.8) together with (7.13) it follows that

$$
A_{\perp}^{a}=-\frac{1}{2} \bar{\eta}^{a b c}\left(e_{b} \mid l_{e_{0}} \vartheta_{c}\right),
$$

and

$$
\left.\underline{X}^{a}=-\mathrm{E}_{e_{0}} \vartheta^{a}=-\frac{1}{2}\left[l_{e_{0}} \vartheta^{a}+\vartheta_{b}\left(e^{a}\right\rfloor l_{e_{0}} \vartheta^{b}\right)\right],
$$

and on the other hand we deduce from (7.9)

$$
\left.\left.\left.\underline{A}^{a}=-\frac{1}{2} \bar{\eta}^{a b c}\left[e_{b}\right\rfloor \underline{d} \vartheta_{c}-\frac{1}{2}\left(e_{b}\right\rfloor e_{c}\right\rfloor \underline{d} \vartheta^{d}\right) \vartheta_{d}\right] .
$$

Equations (8.1-4) reproduce (7.4) decomposed into its constitutive parts, i.e. they ensure that the torsion vanish and that the nonlinear connection reduces to the Christoffel symbol. The equations $(8.1-4)$ are equivalent to those $(7.8,9,12,13)$ from which we derived them. In their original form, we see that three of them, namely $(7.8,9,12)$ are constraints, whereas the fourth one (7.13) is an evolution equation. In the usual interpretation of GR, all these conditions are accepted a priori to hold, as a constitutive part of the Riemannian geometrical background. In this paper we will not enter into the discussion of this point and its consequences. We will do it in a work in course. But we point out that the ignorance of the dynamical character of the vanishing of the torsion is a source of open problems, mostly when one attempts to quantize Gravity.

Let us now look at the remaining dynamical equations. Taking the covariant differential of (7.9), it follows $\bar{\eta}_{a b} \wedge \underline{F}^{b}=0$, which gives rise to $\vartheta_{a} \wedge{ }^{\#} \underline{F}^{a}=0$. On the other hand, as pointed out above, (7.8) implies $\left.e_{[a}\right\rfloor \underline{X}_{b]}=0$. From both results, together with definition (6.8), we conclude that $\vartheta_{a} \wedge{ }^{\#} \underline{\mathcal{R}}^{a}=0$. Similarly, from (7.10) we obtain $\left.\left(e_{a}\right\rfloor{ }^{\#} \underline{\mathcal{R}}^{a}\right)=\Lambda$. Making use of these results, (6.22) reduces to

$$
\beta_{5}^{a}=\underline{D}\left(u^{0} X_{\perp}^{a}\right)+u^{0} \underline{\mathcal{R}}^{a} .
$$

It is also easy to prove that (6.29) reduces to

$$
\beta_{3}^{a}=u^{0} \bar{\eta}_{b c}^{a} X_{\perp}^{b} \underline{X}^{c}-u^{0 \#}\left(\underline{D} \underline{X}^{a}\right) .
$$


Substituting these expressions into $(6.32 \mathrm{~d}, \mathrm{f})$ and taking the definition (6.9) into account, we obtain

$$
\mathrm{E}_{e_{0}} \underline{X}^{a}-\frac{1}{u^{0}} \underline{D}\left(u^{0} X_{\perp}^{a}\right)={ }^{\#} \underline{\mathcal{R}}^{a},
$$

and

$$
\mathcal{R}_{\perp}^{a}=-{ }^{\#}\left(\underline{D} \underline{X}^{a}\right) .
$$

In addition, we have eqs.(7.10) and (7.18), namely

$$
\vartheta_{a} \wedge \underline{\mathcal{R}}^{a}=\Lambda \bar{\eta}
$$

and

$$
\vartheta_{a} \wedge \mathcal{R}_{\perp}^{a}=0 .
$$

Eq.(8.10) is the trace of (8.8) with the constraint (6.31) taken into account. This completes the dynamical information derived from section 6 . What is important to be noticed here is that, whereas (8.10) is present in the Lagrangian equations, (8.8) is not. It is a further restriction to be added to the standard Einstein theory.

Eqs. $(8.7,8)$ represent the time evolution of the tangential parts $\underline{X}^{a}=-\mathrm{E}_{e_{0}} \vartheta^{a}$ of the boost vector and $\underline{A}^{a}$ of the $S O(3)$ connection, see (6.9), respectively. Let us put them together into a fourdimensional formula in order to show them in their simplest form. According to (3.16b), we have

$$
{ }^{*} \mathcal{R}^{a}=\vartheta^{0} \wedge{ }^{\#} \underline{\mathcal{R}}^{a}-{ }^{\#} \mathcal{R}_{\perp}^{a},
$$

and on the other hand, see (3.19),

$$
D X^{a}=\vartheta^{0} \wedge\left[\mathrm{E}_{e_{0}} \underline{X}^{a}-\frac{1}{u^{0}} \underline{D}\left(u^{0} X_{\perp}^{a}\right)\right]+\underline{D} \underline{X}^{a} .
$$

In view of $(8.11,12)$, eq.(8.7) and the dual of (8.8) rearrange into

$$
D X^{a}-{ }^{*} \mathcal{R}^{a}=0
$$

Analogously, (8.9) together with (8.10) take the fourdimensional form

$$
\vartheta_{a} \wedge \mathcal{R}^{a}-\Lambda \eta_{0}=0
$$

being $\left.\eta_{0}:=e_{0}\right\rfloor \eta$, see (E.1). Since the torsion vanishes according to $(8.1-4)$, eqs. $(8.13,14)$ are the condensed form of the Hamiltonian Einstein equations on a Riemannian spacetime.

\section{Relationship with Ashtekar variables}


Finally, let us briefly discuss how our variables relate to those of Ashtekar ${ }^{(9,16)}$. The Ashtekar description derives from the usual $S O(3)$ ADM scheme with dynamical variables $\left(E_{a}{ }^{i}, K_{i}{ }^{a}\right)$ through a quasi Legendre transformation of the form

$$
\begin{aligned}
& E_{a}{ }^{i} \longrightarrow E_{a}{ }^{i} \\
& K_{i}{ }^{a} \longrightarrow A_{i}{ }^{a}=\Gamma_{i}{ }^{a}+\beta{K_{i}}^{a},
\end{aligned}
$$

where $\Gamma_{i}{ }^{a}$ is an $S O(3)$ connection compatible with $E_{a}{ }^{i}$, and $\beta$ is a constant to be fixed later. In this way, the Gauss constraint becomes

$$
\nabla_{i} E_{a}^{i}=0
$$

being the covariant derivative $\nabla_{i}$ constructed in terms of the $S O(3)$ connection $A_{i}{ }^{a}$. The vectorial constraint takes the form

$$
F_{i j}{ }^{a} E_{a}^{j}=0
$$

with $F_{i j}{ }^{a}$ as the usual $S O(3)$ field strength tensor. Finally, the scalar constraint becomes

$$
-\zeta \epsilon_{a b c} E^{a}{ }_{i} E^{b}{ }_{j} F^{i j c}+2\left(\zeta-\frac{1}{\beta^{2}}\right) E_{\left[a^{i}\right.} E_{b]}{ }^{j}\left(A_{i}{ }^{a}-\Gamma_{i}{ }^{a}\right)\left(A_{j}{ }^{b}-\Gamma_{j}{ }^{b}\right)=0,
$$

where $\zeta$ stands for the signature, corresponding in particular $\zeta=-1$ to the Lorentzian one considered by us.

Let us now compare (9.2-4) with our results. In order to do so, we will express the relevant equations obtained in section 6 in terms of components, making use of the notation

$$
\vartheta^{a}=e^{a}{ }_{i} d x^{i} \quad, \quad \underline{X}^{a}=\underline{X}_{i}{ }^{a} d x^{i} \quad, \quad \underline{A}^{a}=\underline{A}_{i}{ }^{a} d x^{i} .
$$

The constraint $(6.19 \mathrm{~d})$ gives rise to

$$
\#\left(\underline{D} \bar{\eta}_{a}\right)=\underline{D}_{i} e_{a}^{i}=0,
$$

which coincides with the Gauss constraint (9.2) if we identify $e^{a}{ }_{i}$ with $E^{a}{ }_{i}$. On the other hand, taking the covariant differential of $(6.23 \mathrm{~d})$ we get

$$
\underline{D} \wedge \underline{D} \vartheta^{a} \equiv \bar{\eta}_{b}^{a} \wedge \underline{F}^{b}=0 .
$$

From (9.7) follows the vectorial costraint

$$
e_{i}^{a} \#\left(\bar{\eta}_{a b} \wedge \underline{F}^{b}\right)=\underline{F}_{i j}{ }^{a} e_{a}^{j}=0,
$$


compare with (9.3). Finally, we develop our scalar constraint (6.23a) as

$$
\#\left(\vartheta_{a} \wedge \underline{\mathcal{R}}^{a}-\Lambda \bar{\eta}\right)=\frac{1}{2}\left(\epsilon_{a b c} e^{a}{ }_{i} e^{b}{ }_{j} \underline{F}^{i j c}-2 e_{[a}{ }^{i} e_{b]}{ }^{j} \underline{X}_{i}^{a} \underline{X}_{j}{ }^{b}\right)-\Lambda=0 .
$$

Taking $\underline{X}_{i}^{a}$ proportional to $\left(A_{i}{ }^{a}-\Gamma_{i}{ }^{a}\right)$, eq.(9.9) with $\Lambda=0$ coincides with (9.4), with the Lorentzian signature $\zeta=-1$, in the limit $\beta=1$ suggested by Barbero ${ }^{(16)}$. Furthermore, the standard complex Ashtekar variables corresponding to the choice $\beta=i$ also relate to ours in a simple way as follows. Let us perform the transformation

$$
\underline{A}^{a} \longrightarrow \underline{\tilde{A}}^{a}=\underline{A}^{a}+i \underline{X}^{a} \text {. }
$$

The field strength constructed with the complex $S O(3)$ connection $\underline{\tilde{A}}^{a}$, in terms of the original real variables reads

$$
\underline{\tilde{F}}^{a}=\underline{\mathcal{R}}^{a}+i \underline{D} \underline{X}^{a},
$$

see $(6.8)$. Taking now the constraints $(7.8,9,11)$ into account, we verify that

$$
\underline{D} \bar{\eta}_{a}=\underline{\tilde{D}} \bar{\eta}_{a}, \quad \bar{\eta}_{a b} \wedge \underline{F}^{b}=\bar{\eta}_{a b} \wedge \underline{\tilde{E}}^{b}, \quad \vartheta_{a} \wedge \underline{\mathcal{R}}^{a}=\vartheta_{a} \wedge \underline{\tilde{F}}^{a} .
$$

The expressions with tilde depend on the complex connection, see (9.10). In terms of them, the constraints $(9.6,8,9)$ take the usual Ashtekar form, namely

$$
\begin{gathered}
\#\left(\underline{D} \bar{\eta}_{a}\right)=\underline{\tilde{D}}_{i} e_{a}{ }^{i}=0, \\
e^{a}{ }_{i} \#\left(\bar{\eta}_{a b} \wedge \underline{F}^{b}\right)=\underline{\tilde{F}}_{i j}{ }^{a} e_{a}{ }^{j}=0,
\end{gathered}
$$

and

$$
\#\left(\vartheta_{a} \wedge \underline{\mathcal{R}}^{a}-\Lambda \bar{\eta}\right)=\frac{1}{2} \epsilon_{a b c} e^{a}{ }_{i} e^{b}{ }_{j} \underline{\tilde{F}}^{i j c}-\Lambda=0 .
$$

We point out that the physical meaning of the change (9.1) in the Ashtekar approach becomes evident in relation with the dynamics of the $X^{a}$ vectors associated to the boosts.

\section{Conclusions}

Taking advantage of the fact that a particular nonlinear realization of the Poincaré group defines a natural time direction, we performed a Poincaré invariant spacetime foliation, and we constructed a Hamiltonian formalism adapted to the local spacetime symmetry. We identified the gravitational dynamical variables to be nonlinear connections (differential 1-forms) with $S O(3)$ indices corresponding to the classification subgroup. 
From the Hamiltonian evolution equations corresponding to the Einstein-Cartan action we reproduced the standard Lagrangian field equations of GR, but we also proved that the Hamiltonian ones are more restrictive, and we obtained the complete set of constraints. Ashtekar variables were identified with the natural dynamical coordinates of our nonlinear Hamiltonian description of Gravity.

It would be interesting to study more general actions. In fact, as a consequence of the nonlinear approach, a large number of Poincaré invariants exist with respect to the explicit $S O(3)$ classification subgroup, which are not expressible in the fourdimensional geometrical language, and accordingly we have at our disposal additional invariant terms which make more flexible the choice of gravitational actions.

\section{Acknowledgements}

We cordially thank Prof. Manuel de León for his valuable suggestions and help in several hard mathematical questions, Dr. Fernando Barbero for his contribution to our understanding of the link between the nonlinear gauge fields and Ashtekar variables, and Prof. Friedrich Wilhelm Hehl for advising us on his transparent treatment of gravitational gauge theories, and for constant interest in our work.

\section{APPENDICES}

\section{A.-Coset realizations of symmetry groups}

In this appendix we briefly summarize the nonlinear coset realization procedure ${ }^{(4)}$ which constitutes the basis of the particular application of section 2 and thus of the whole present work.

Let $G=\{g\}$ be a Lie group including a subgroup $H=\{h\}$ whose linear representations $\rho(h)$ are known, acting on functions $\psi$ belonging to a linear representation space of $H$. The elements of the quotient space $G / H$ are equivalence classes of the form $g H=\left\{g h_{1}, g h_{2} \ldots g h_{n}\right\}$, and they constitute a complete partition of the group space. We call the elements of the quotient space cosets to the left (right) of $G$ with respect to $H$. Since we deal with Lie groups, the elements of $G / H$ are labeled by continuous parameters, say $\xi$. We represent the elements of $G / H$ by means of the coset indicators $c(\xi)$, parametrized by the coset parameters $\xi$, playing the role of a kind of coordinates. The nonlinear coset realizations are based on the action of the group on $G / H$, i.e., on a partition of its own space. An arbitrary element $g \epsilon G$ acts on $G / H$ transforming a coset into another, that is

$$
\begin{aligned}
g: G / H & \rightarrow G / H \\
c(\xi) & \rightarrow c\left(\xi^{\prime}\right),
\end{aligned}
$$


according to the general law

$$
g c(\xi)=c\left(\xi^{\prime}\right) h(\xi, g) .
$$

The elements $h(\xi, g)$ which appear in (A.2) belong to the subgroup $H$, that we will call in the following the classification subgroup, since the elements $g$ of the whole group $G$ considered in (A.2) act nonlinearly on the representation space of the classification subgroup $H$ according to

$$
\psi^{\prime}=\rho(h(\xi, g)) \psi,
$$

where $\rho$, as mentioned above, is a linear representation of $H$ in the space of the matter fields $\psi$. Therefore, the action of the total group $G$ projects on the representations of the subgroup $H$ through the dependence of $h(\xi, g)$ in (A.2) on the group element $g$, as given by eq.(A.3). The action of the group is realized on the couples $(\xi, \psi)$. It reduces to the usual linear action of $H$ when we take in particular for $g$ in (A.2) an element of $H$.

In order to define a covariant differential transforming like (A.3) under local transformations, we need a suitable nonlinear connection. We define it as

$$
\Gamma:=c^{-1} \mathcal{D} c,
$$

where the covariant differential on the coset space is defined as

$$
\mathcal{D} c:=(d+\Omega) c,
$$

with the ordinary linear connection $\Omega$ of the whole group $\mathrm{G}$ transforming as

$$
\Omega^{\prime}=g \Omega g^{-1}+g d g^{-1} .
$$

It is easy to prove that the nonlinear gauge field $\Gamma$ defined in (A.4) transforms as

$$
\Gamma^{\prime}=h \Gamma h^{-1}+h d h^{-1},
$$

thus allowing to define the nonlinear covariant differential operator

$$
\mathbf{D}:=d+\Gamma .
$$

One can read out from (A.7) that only the components of $\Gamma$ related to the generators of $H$ behave as true connections, transforming inhomogeneously, whereas the components of $\Gamma$ over the generators associated with the cosets $c$ transform as tensors with respect to the subgroup $H$ notwithstanding their nature of connections.

\section{B.-The Poincaré group in terms of boosts, rotations and space and time translations}


The calculations leading to the results of section 2 rest on the decomposition of the generators of the Poincare group presented in this Appendix. In particular, we made use of the commutation relations (B.6).

In the fourdimensional notation, the Lorentz generators $L_{\alpha \beta}$ and the translational generators $P_{\alpha} \quad(\alpha, \beta=0 \ldots 3)$ of the Poincaré group satisfy the commutation relations

$$
\begin{aligned}
{\left[L_{\alpha \beta}, L_{\mu \nu}\right] } & =-i\left(o_{\alpha[\mu} L_{\nu] \beta}-o_{\beta[\mu} L_{\nu] \alpha}\right) \\
{\left[L_{\alpha \beta}, P_{\mu}\right] } & =i o_{\mu[\alpha} P_{\beta]}, \\
{\left[P_{\alpha}, P_{\beta}\right] } & =0 .
\end{aligned}
$$

We choose the invariant metric tensor to be

$$
o_{\alpha \beta}:=\operatorname{diag}(-+++) .
$$

We can decompose the generators in such a way that

$$
\beta^{\alpha \beta} L_{\alpha \beta}=2 \beta^{a 0} L_{a 0}+\beta^{a b} L_{a b},
$$

with $a, b$ running from 1 to 3 . Let us define

$$
\begin{gathered}
S_{a}:=-\epsilon_{a}^{b c} L_{b c}, \\
K_{a}:=2 L_{a 0} .
\end{gathered}
$$

The generators (B.4) are those of the $S O(3)$ group, and (B.5) correspond to the boosts. In terms of them, and taking (B.2) into account, the commutation relations (B.1) transform into

$$
\begin{aligned}
{\left[S_{a}, S_{b}\right] } & =-i \epsilon_{a b c} S_{c}, \\
{\left[K_{a}, K_{b}\right] } & =i \epsilon_{a b c} S_{c}, \\
{\left[S_{a}, K_{b}\right] } & =-i \epsilon_{a b c} K_{c}, \\
{\left[S_{a}, P_{0}\right] } & =0 \\
{\left[S_{a}, P_{b}\right] } & =-i \epsilon_{a b c} P_{c}, \\
{\left[K_{a}, P_{0}\right] } & =i P_{a}, \\
{\left[K_{a}, P_{b}\right] } & =i \delta_{a b} P_{0}, \\
{\left[P_{a}, P_{b}\right] } & =\left[P_{a}, P_{0}\right]=\left[P_{0}, P_{0}\right]=0 .
\end{aligned}
$$

Thus we can rewrite (B.3) as

$$
\beta^{\alpha \beta} L_{\alpha \beta}=\xi^{a} K_{a}+\theta^{a} S_{a},
$$


with the new coefficients used in section 2 defined in an obvious way.

The following well known formulae will be useful for the audacious reader who wants to reproduce the calculations.

$$
\begin{gathered}
\epsilon_{a b c} \epsilon^{m n s}=2\left(\delta_{[a}^{m} \delta_{b]}^{n} \delta_{c}^{s}+\delta_{[a}^{s} \delta_{b]}^{m} \delta_{c}^{n}+\delta_{[a}^{n} \delta_{b]}^{s} \delta_{c}^{m}\right) \\
\epsilon_{a b c} \epsilon^{m n c}=2 \delta_{[a}^{m} \delta_{b]}^{n}, \\
\epsilon_{a b c} \epsilon^{m b c}=2 \delta_{a}^{m}, \\
\epsilon_{a b c} \epsilon^{a b c}=3 ! \\
e^{-A} B e^{A}=B-[A, B]+\frac{1}{2 !}[A,[A, B]]-\ldots \\
e^{-\chi A} d e^{\chi A}=d \chi A-\frac{1}{2 !}[\chi A, d \chi A]+\frac{1}{3 !}[\chi A,[\chi A, d \chi A]]-\ldots \\
e^{i\left(\lambda^{a}+\delta \lambda^{a}\right) K_{a}}=e^{i \lambda^{a} K_{a}}\left(1+e^{-i \lambda^{a} K_{a}} \delta e^{i \lambda^{a} K_{a}}\right) .
\end{gathered}
$$

The choice of signs in $(2.11,12)$ is conventional. We wanted to be consistent with our previous work ${ }^{(3)}$ and at the same time we attempted to reproduce the usual sign conventions in the definition of $S O(3)$ covariant differentials and curvature.

\section{C.-The Hamiltonian formalism}

Here we present an alternative deduction of the Hamiltonian formalism. We will not pay attention to the constraints since we are only interested in showing that the Lie derivatives obtained in the abbreviated way of section 4 coincide with those derived in the standard approach sketched in this Appendix. Let us consider a Lagrangian density

$$
L=L\left(u^{0}, d u^{0}, \alpha, d \alpha\right),
$$

being $\alpha$ a p-form. We include in (C.1) the explicit dependence on $u^{0}$ in order to take into account the applicability of the formalism to Gravity, as it is done in section 6. In the absence of a gravitational action, $u^{0}$ belongs to the geometrical background and does not play any dynamical role. We decompose the variables $\alpha$ into their normal

and tangential parts, see $(3.16,19)$, and accordingly we rewrite the Lagrangian density as (4.3), with

$$
L_{\perp}=L_{\perp}\left(u^{0}, \underline{d} u^{0}, \alpha_{\perp}, \underline{\alpha}, \underline{d} \alpha_{\perp}, \underline{d} \underline{\alpha}, l_{e_{0}} \underline{\alpha}\right) .
$$


The variation

$$
\begin{aligned}
\delta L=\vartheta^{0} \wedge\left\{\delta u^{0}\left[\frac{\partial L_{\perp}}{\partial u^{0}}-\frac{1}{u^{0}} \underline{d}\left(u^{0} \frac{\partial L_{\perp}}{\partial\left(\underline{d} u^{0}\right)}\right)-\frac{1}{u^{0}}\left(l_{e_{0}} \underline{\alpha} \wedge \frac{\partial L_{\perp}}{\partial\left(l_{e_{0}} \underline{\alpha}\right)}-L_{\perp}\right)\right]\right. \\
+\delta \alpha_{\perp} \wedge\left[\frac{\partial L_{\perp}}{\partial \alpha_{\perp}}+(-1)^{p} \frac{1}{u^{0}} \underline{d}\left(u^{0} \frac{\partial L_{\perp}}{\partial\left(\underline{d} \alpha_{\perp}\right)}\right)\right] \\
\left.+\delta \underline{\alpha} \wedge\left[\frac{\partial L_{\perp}}{\partial \underline{\alpha}}-(-1)^{p} \frac{1}{u^{0}} \underline{d}\left(u^{0} \frac{\partial L_{\perp}}{\partial(\underline{d} \underline{\alpha})}\right)-l_{e_{0}}\left(\frac{\partial L_{\perp}}{\partial\left(l_{e_{0}} \underline{\alpha}\right)}\right)\right]\right\} \\
+d\left\{\delta \underline{\alpha} \wedge \frac{\partial L_{\perp}}{\partial\left(l_{e_{0}} \underline{\alpha}\right)}-\vartheta^{0} \wedge\left[\delta u^{0} \frac{\partial L_{\perp}}{\partial\left(\underline{d} u^{0}\right)}+\delta \alpha_{\perp} \wedge \frac{\partial L_{\perp}}{\partial\left(\underline{d} \alpha_{\perp}\right)}+\delta \underline{\alpha} \wedge \frac{\partial L_{\perp}}{\partial(\underline{d} \underline{\alpha})}\right]\right\}
\end{aligned}
$$

gives rise to the field equations

$$
\begin{aligned}
& \frac{\partial L_{\perp}}{\partial u^{0}}-\frac{1}{u^{0}} \underline{d}\left(u^{0} \frac{\partial L_{\perp}}{\partial\left(\underline{d} u^{0}\right)}\right)-\frac{1}{u^{0}}\left(l_{e_{0}} \underline{\alpha} \wedge \frac{\partial L_{\perp}}{\partial\left(l_{e_{0}} \underline{\alpha}\right)}-L_{\perp}\right)=0 \\
& \frac{\partial L_{\perp}}{\partial \alpha_{\perp}}+(-1)^{p} \frac{1}{u^{0}} \underline{d}\left(u^{0} \frac{\partial L_{\perp}}{\partial\left(\underline{d} \alpha_{\perp}\right)}\right)=0 \\
& \frac{\partial L_{\perp}}{\partial \underline{\alpha}}-(-1)^{p} \frac{1}{u^{0}} \underline{d}\left(u^{0} \frac{\partial L_{\perp}}{\partial(\underline{d} \underline{\alpha})}\right)-l_{e_{0}}\left(\frac{\partial L_{\perp}}{\partial\left(l_{e_{0}} \underline{\alpha}\right)}\right)=0 .
\end{aligned}
$$

From (C.2) we define the only nonvanishing momentum (remember that we will not study the constraints at this stage)

$$
{ }^{\#} \pi:=\frac{\partial L_{\perp}}{\partial\left(l_{e_{0}} \underline{\alpha}\right)},
$$

and we define the Hamiltonian

$$
\mathcal{H}:=u^{0}\left(l_{e_{0}} \underline{\alpha} \wedge^{\#} \pi-L_{\perp}\right) .
$$

The variation of (C.6) reads

$$
\begin{aligned}
& \delta \mathcal{H}=u^{0}\{ l_{e_{0}} \underline{\alpha} \wedge \delta^{\#} \pi \\
&-\delta u^{0}\left[\frac{\partial L_{\perp}}{\partial u^{0}}-\frac{1}{u^{0}} \underline{d}\left(u^{0} \frac{\partial L_{\perp}}{\partial\left(\underline{d} u^{0}\right)}\right)-\frac{1}{u^{0}}\left(l_{e_{0}} \underline{\alpha} \wedge{ }^{\#} \pi L_{\perp}\right)\right] \\
&-\delta \alpha_{\perp} \wedge\left[\frac{\partial L_{\perp}}{\partial \alpha_{\perp}}+(-1)^{p} \frac{1}{u^{0}} \underline{d}\left(u^{0} \frac{\partial L_{\perp}}{\partial\left(\underline{d} \alpha_{\perp}\right)}\right)\right] \\
&\left.-\delta \underline{\alpha} \wedge\left[\frac{\partial L_{\perp}}{\partial \underline{\alpha}}-(-1)^{p} \frac{1}{u^{0}} \underline{d}\left(u^{0} \frac{\partial L_{\perp}}{\partial(\underline{d} \underline{\alpha})}\right)\right]\right\} \\
&-\underline{d}\left\{u^{0}\left[\delta u^{0} \frac{\partial L_{\perp}}{\partial\left(\underline{d} u^{0}\right)}+\delta \alpha_{\perp} \wedge \frac{\partial L_{\perp}}{\partial\left(\underline{d} \alpha_{\perp}\right)}+\delta \underline{\alpha} \wedge \frac{\partial L_{\perp}}{\partial(\underline{d} \underline{\alpha})}\right]\right\} .
\end{aligned}
$$


Making use of the field equations (C.4) and neglecting the total divergence contributions, (C.7) transforms into

$$
\delta \mathcal{H}=u^{0}\left[l_{e_{0}} \underline{\alpha} \wedge \delta^{\#} \pi-\delta \underline{\alpha} \wedge l_{e_{0}}\left(\frac{\partial L_{\perp}}{\partial\left(l_{e_{0}} \underline{\alpha}\right)}\right)\right]=u^{0}\left[l_{e_{0}} \underline{\alpha} \wedge \delta^{\#} \pi-\delta \underline{\alpha} \wedge l_{e_{0}}^{\#} \pi\right]
$$

On the other hand, being

$$
\mathcal{H}=\mathcal{H}\left(u^{0}, \alpha_{\perp}, \underline{\alpha},{ }^{\#} \pi\right)
$$

we find alternatively

$$
\delta \mathcal{H}=\delta u^{0} \frac{\delta \mathcal{H}}{\delta u^{0}}+\delta \alpha_{\perp} \wedge \frac{\delta \mathcal{H}}{\delta \alpha_{\perp}}+\delta \underline{\alpha} \wedge \frac{\delta \mathcal{H}}{\delta \underline{\alpha}}+\frac{\delta \mathcal{H}}{\delta \# \pi} \wedge \delta^{\#} \pi
$$

Comparing (C.8) with (C.10), the Hamilton equations follow, namely

$$
\frac{\delta \mathcal{H}}{\delta u^{0}}=0 \quad, \quad \frac{\delta \mathcal{H}}{\delta \alpha_{\perp}}=0
$$

and

$$
u^{0} l_{e_{0}} \underline{\alpha}=\frac{\delta \mathcal{H}}{\delta \# \pi} \quad, \quad u^{0} l_{e_{0}}^{\#} \pi=-\frac{\delta \mathcal{H}}{\delta \underline{\alpha}}
$$

Eqs.(C.11) have to do with the existence of constraints and are treated more rigorously in the text. The important thing we wanted to demonstrate here is that (C.12) coincide with (4.8) as deduced shortly in section 4 .

\section{D.-Poisson brackets}

We denote the canonically conjugated variables in a compact form as $Q_{i}^{a},{ }^{\#} \Pi_{b}^{i}$. Let $\omega$ be a $\mathrm{p}$-form and $\sigma$ a $\mathrm{q}$-form depending on these variables. We generalize the definition (4.10) of the Poisson brackets in the obvious form

$$
\begin{aligned}
\{\omega, \sigma\} & :=\frac{\delta \sigma}{\delta \# \Pi_{a}^{i}} \wedge \frac{\delta \omega}{\delta Q_{i}^{a}}-\frac{\delta \omega}{\delta \# \Pi_{a}^{i}} \wedge \frac{\delta \sigma}{\delta Q_{i}^{a}} \\
& =\left\{Q_{i}^{a}, \sigma\right\} \wedge \frac{\delta \omega}{\delta Q_{i}^{a}}+\frac{\delta \omega}{\delta \# \Pi_{a}^{i}} \wedge\left\{\# \Pi_{a}^{i}, \sigma\right\}
\end{aligned}
$$

It follows

$$
\begin{gathered}
\{\omega, \sigma\}=-\{\sigma, \omega\} \\
\{\omega \wedge \alpha, \sigma\}=\{\omega, \sigma\} \wedge \alpha+(-1)^{p(q+1)} \omega \wedge\{\alpha, \sigma\} .
\end{gathered}
$$


In particular, the fundamental Poisson brackets satisfy

$$
\begin{aligned}
\left\{Q_{i}^{a}, Q_{j}^{b}\right\} & =0 \\
\left\{{ }^{\#} \Pi_{a}^{i},{ }^{\#} \Pi_{b}^{j}\right\} & =0 \\
\left\{Q_{i}^{a}(x),{ }^{\#} \Pi_{b}^{j}(y)\right\} & =\delta_{b}^{a} \delta_{i}^{j} \delta(x-y),
\end{aligned}
$$

and in addition

$$
\left\{\underline{D} Q_{i}^{a},{ }^{\#} \Pi_{b}^{j}\right\}=\underline{D}\left\{Q_{i}^{a},{ }^{\#} \Pi_{b}^{j}\right\} .
$$

Being $Q_{i}^{a}$ not a vector but a connection $\underline{A}^{a}$, we find

$$
\left\{\underline{F}^{a},{ }^{\#} \Pi \underline{A}\right\}=\left\{\underline{A}^{a}, \underline{D}^{\#} \Pi \underline{A}\right\}=\underline{D}\left\{A^{a},{ }^{\#} \Pi \underline{A}\right\} .
$$

On the other hand, being $\varphi_{a}^{(1)}$, see $(6.19 \mathrm{~b})$, a first class constraint, it is the generator of a symmetry, namely of the rotations. In fact, for any vector-valued form $\omega^{a}$ holds

$$
\left\{\omega^{a}, \varphi_{b}^{(1)}\right\}=\epsilon_{b c}^{a} \omega^{c},
$$

so that

$$
\delta \omega^{a}=\lambda^{b}\left\{\omega^{a}, \varphi_{b}^{(1)}\right\}=\epsilon_{b c}^{a} \lambda^{b} \omega^{c},
$$

whereas for the $S O(3)$ connection we find

$$
\delta \underline{A}^{a}=\lambda^{b}\left\{\underline{A}^{a}, \varphi_{b}^{(1)}\right\}=-\underline{D} \lambda^{b} .
$$

Thus, from the relation

$$
\delta \underline{D} \omega^{a}=\underline{D} \delta \omega^{a}+\epsilon_{b c}^{a} \delta A^{b} \wedge \omega^{c},
$$

we find

$$
\left\{\underline{D} \omega^{a}, \varphi_{b}^{(1)}\right\}=\underline{D}\left\{\omega^{a}, \varphi_{b}^{(1)}\right\} .
$$

This and similar properties were used in the calculation of the constraints and of their stability conditions.

\section{E.-Several useful formulae}

The Hodge dual star in three dimensions is defined as follows

$$
\begin{aligned}
\#\left(\vartheta_{a} \wedge \vartheta_{b} \wedge \vartheta_{c}\right) & \left.:=\bar{\eta}_{a b c}:=\eta_{0 a b c}=-e_{0}\right\rfloor \eta_{a b c} \\
\#\left(\vartheta_{a} \wedge \vartheta_{b}\right) & \left.:=\bar{\eta}_{a b}:=\bar{\eta}_{a b c} \vartheta^{c}=e_{0}\right\rfloor \eta_{a b} \\
\# \vartheta_{a} & \left.:=\bar{\eta}_{a}:=\frac{1}{2 !} \bar{\eta}_{a b c} \vartheta^{b} \wedge \vartheta^{c}=-e_{0}\right\rfloor \eta_{a} \\
\# 1 & \left.:=\bar{\eta}:=\frac{1}{3 !} \bar{\eta}_{a b c} \vartheta^{a} \wedge \vartheta^{b} \wedge \vartheta^{c}=e_{0}\right\rfloor \eta
\end{aligned}
$$


The variation of dual forms reads, see Ref.(13),

$$
\left.\left.\delta^{*} \alpha={ }^{*} \delta \alpha-{ }^{*}\left(\delta \vartheta^{\alpha} \wedge e_{\alpha}\right\rfloor \alpha\right)+\delta \vartheta^{\alpha} \wedge\left(e_{\alpha}\right\rfloor^{*} \alpha\right)
$$

Making use of the relations

$$
{ }^{\#} \alpha_{\perp}=-{ }^{*}\left(\vartheta^{0} \wedge \alpha_{\perp}\right), \quad{ }^{\#} \underline{\alpha}=-{ }^{*}\left(\vartheta^{0} \wedge \alpha\right)
$$

we find

$$
\begin{gathered}
\left.\left.\delta^{\#} \alpha_{\perp}={ }^{\#} \delta \alpha_{\perp}-{ }^{\#}\left(\delta \vartheta^{a} \wedge e_{a}\right\rfloor \alpha_{\perp}\right)+\delta \vartheta^{a} \wedge\left(e_{a}\right\rfloor^{\#} \alpha_{\perp}\right) \\
\left.\left.\delta^{\#} \underline{\alpha}={ }^{\#} \delta \underline{\alpha}-{ }^{\#}\left(\delta \vartheta^{a} \wedge e_{a}\right\rfloor \underline{\alpha}\right)+\delta \vartheta^{a} \wedge\left(e_{a}\right\rfloor^{\#} \underline{\alpha}\right) .
\end{gathered}
$$

Other formulae necessary to reproduce the calculations of this paper are the following $(6,7,12)$

$$
\begin{gathered}
{ }^{\#} \alpha=\alpha, \\
\left.\#\left(\alpha \wedge \vartheta_{\alpha}\right)=e_{\alpha}\right\rfloor^{\#} \alpha .
\end{gathered}
$$

Being $\alpha$ and $\beta \mathrm{p}$-forms

$$
\begin{aligned}
& { }^{\#} \alpha \wedge \beta={ }^{\#} \beta \wedge \alpha, \\
& \left.\vartheta^{a} \wedge e_{a}\right\rfloor \alpha=p \alpha .
\end{aligned}
$$

\section{REFERENCES}

[1] R. Utiyama, Phys. Rev. 101 (1956) 1597

T. W. B. Kibble, J. Math. Phys. 2 (1961) 212

D. W. Sciama, Rev. Mod. Phys. 36 (1964) 463 and 1103

A. Trautman, in Differential Geometry, Symposia Mathematica Vol. 12 (Academic Press, London, 1973), p. 139

A. G. Agnese and P. Calvini, Phys. Rev. D 12 (1975) 3800 and 3804

P. von der Heyde, Phys. Lett. 58 A (1976) 141

E.A. Ivanov and J. Niederle, Phys. Rev. D25 (1982) 976 and 988

D. Ivanenko and G.A. Sardanashvily, Phys. Rep. 94 (1983) 1

E. A. Lord, J. Math. Phys. 27 (1986) 2415 and 3051

[2] A.B. Borisov and I.V. Polubarinov, Zh. ksp. Theor. Fiz. 48 (1965) 1625, and V. Ogievetsky and I. Polubarinov, Ann. Phys. (NY) 35 (1965) 167

A.B. Borisov and V.I. Ogievetskii, Theor. Mat. Fiz. 21 (1974) 329

A. Salam and J. Strathdee, Phys. Rev. 184 (1969) 1750 and 1760 
C.J. Isham, A. Salam and J. Strathdee, Ann. of Phys. 62 (1971) 98

L.N. Chang and F. Mansouri, Phys. Lett. 78 B (1979) 274, and Phys. Rev. D 17 (1978) 3168

K.S. Stelle and P.C. West, Phys. Rev. D 21 (1980) 1466

E.A. Lord, Gen. Rel. Grav. 19 (1987) 983, and J. Math. Phys. 29 (1988) 258

[3] A. López-Pinto, A. Tiemblo and R. Tresguerres, Class. Quantum Grav. 12 (1995) 1327

[4] S. Coleman, J. Wess and B. Zumino, Phys. Rev. 117 (1969) 2239

C.G. Callan, S. Coleman, J. Wess and B. Zumino, Phys. Rev. 117 (1969) 2247

S. Coleman, Aspects of Symmetry. Cambridge University Press, Cambridge (1985)

[5] K. Hayashi and T. Nakano, Prog. Theor. Phys 38 (1967) 491

K. Hayashi and T. Shirafuji, Prog. Theor. Phys 64 (1980) 866 and 80 (1988) 711

G. Grignani and G. Nardelli, Phys. Rev. D 45 (1992) 2719

E. W. Mielke, J.D. McCrea, Y. Ne'eman and F.W. Hehl Phys. Rev. D 48 (1993) 673 , and references therein

J. Julve, A. López-Pinto, A. Tiemblo and R. Tresguerres, Nonlinear gauge realizations of spacetime symmetries including translations, to appear in G.R.G. (1996)

[6] F. W. Hehl, J. D. McCrea, E. W. Mielke, and Y. Ne'eman Found. Phys. 19 (1989) 1075

R. D. Hecht and F. W. Hehl, Proc. 9th Italian Conf. G.R. and Grav. Phys., Capri (Napoli). R. Cianci et al.(eds.) (World Scientific, Singapore, 1991) p. 246

F.W. Hehl, J.D. McCrea, E.W. Mielke, and Y. Ne'eman, Physics Reports 258 (1995) 1

[7] F.W. Hehl, P. von der Heyde, G. D. Kerlick and J. M. Nester, Rev. Mod. Phys. 48 (1976) 393

F.W. Hehl, Proc. of the 6th Course of the School of Cosmology and Gravitation on Spin, Torsion, Rotation, and Supergravity, held at Erice, Italy, May 1979, P.G. Bergmann, V. de Sabbata, eds. (Plenum, N.Y. i980) 5

[8] P. Baekler, F.W. Hehl, and E.W. Mielke, Proc. of the 2nd Marcel Grossmann Meeting on Recent Progress of the Fundamentals of General Relativity 1978, R. Ruffini, ed. (North-Holland, Amsterdam 1982) 413

P. Baekler, F.W. Hehl, and H.J. Lenzen, Proc. of the 3rd Marcel Grossmann Meeting on General Relativity, Hu Ning, ed. (North-Holland, Amsterdam 1983) 107 
J.D. McCrea, P. Baekler and M. Gürses, Nuovo Cimento B 99 (1987) 171

P. Baekler, M. Gürses, F.W. Hehl and J.D. McCrea, Phys. Lett. A 128 (1988) 245

R. Tresguerres, Z. Phys. C 65 (1995) 347, and Phys. Lett. A 200 (1995) 405

[9] A. Ashtekar, Phys. Rev. Lett. 57 (1986) 2244

A. Ashtekar, Phys. Rev. D 36 (1987) 1587

A. Ashtekar, Non Perturbative Canonical Gravity (Notes prepared in collaboration with R. S. Tate). (World Scientific Books, Singapore, 1991)

[10] P.A.M. Dirac, Canadian Jour. of Math. 2 (1950) 129

P.A.M. Dirac, Lectures on Quantum Mechanics, Belfer Graduate School of Science, Yeshiva University, N.Y. (1964)

[11] A. Hanson, T. Regge and C. Teitelboim, Constrained Hamiltonian Systems, Roma, Accademia dei Lincei (1976)

[12] E.W. Mielke, Phys. Lett. A 149 (1990) 345

E.W. Mielke, Ann. Phys. (N.Y.) 219 (1992) 78

[13] R.P. Wallner, Ph.D. Thesis, University of Vienna (1982)

R.P. Wallner, Phys. Rev. D 42 (1990) 441

R.P. Wallner, Jour. Math. Phys. 36 (1995) 6937

[14] E.P. Wigner, Ann. Math. 40 (1939) 149

S. Weinberg, Phys. Rev. 133 B (1964) 1318

[15] F.W. Warner, Foundations of differentiable manifolds and Lie groups, Scott, Foresman and Company, Glenview, Illinois (1971)

[16] J. F. Barbero G., Physical Review D 51 (1995) 5507 